\title{
A new family of orthogonal polynomials in three variables
}

\author{
Rabia Aktaş ${ }^{1 *}$ (D), Iván Area² (D) and Esra Güldoğan³ (D)
}

${ }^{*}$ Correspondence:

raktas@science.ankara.edu.tr

'Department of Mathematics,

Faculty of Science, Ankara

University, Ankara, Turkey

Full list of author information is

available at the end of the article

\section{Springer}

\begin{abstract}
In this paper we introduce a six-parameter generalization of the four-parameter three-variable polynomials on the simplex and we investigate the properties of these polynomials. Sparse recurrence relations are derived by using ladder relations for shifted univariate Jacobi polynomials and bivariate polynomials on the triangle. Via these sparse recurrence relations, second order partial differential equations are presented. Some connection relations are obtained between these polynomials. Also, new results for the four-parameter three-variable polynomials on the simplex are given. Finally, some generating functions are derived.
\end{abstract}

MSC: Primary 33C50; secondary 33C45

Keywords: Jacobi polynomials; Koornwinder polynomials; Generating function; Recurrence relation; Partial differential equation; Connection relation

\section{Introduction}

The classical univariate Jacobi polynomials $P_{n}^{(a, b)}(x)$ defined by the Rodrigues formula,

$$
P_{n}^{(a, b)}(x)=\frac{(-1)^{n}}{2^{n} n !}(1-x)^{-a}(1+x)^{-b} \frac{d^{n}}{d x^{n}}\left\{(1-x)^{n+a}(1+x)^{n+b}\right\}, \quad n \geq 0,
$$

are orthogonal with respect to the weight function $w_{a, b}(x)=(1-x)^{a}(1+x)^{b}$ on the interval $(-1,1)$ for $a, b>-1$. The Jacobi polynomials on the interval $(0,1)$, also referred as shifted univariate Jacobi polynomials [8], which we denote by $\widetilde{P}_{n}^{(a, b)}(x):=P_{n}^{(a, b)}(2 x-1)$,

$$
\widetilde{P}_{n}^{(a, b)}(x)=\frac{(-1)^{n}}{n !}(1-x)^{-a} x^{-b} \frac{d^{n}}{d x^{n}}\left\{(1-x)^{n+a} x^{n+b}\right\}, \quad n \geq 0,
$$

are orthogonal on the interval $(0,1)$ with respect to the weight function $\widetilde{w}_{a, b}(x)=(1-x)^{a} x^{b}$ where $a, b>-1$. Indeed, we have

$$
\int_{0}^{1} \widetilde{P}_{n}^{(a, b)}(x) \widetilde{P}_{m}^{(a, b)}(x)(1-x)^{a} x^{b} d x=\delta_{m, n} h_{n}^{(a, b)},
$$

where

$$
h_{n}^{(a, b)}=\frac{\Gamma(a+n+1) \Gamma(b+n+1)}{n !(a+b+2 n+1) \Gamma(a+b+n+1)},
$$

(c) The Author(s) 2020. This article is licensed under a Creative Commons Attribution 4.0 International License, which permits use, sharing, adaptation, distribution and reproduction in any medium or format, as long as you give appropriate credit to the original author(s) and the source, provide a link to the Creative Commons licence, and indicate if changes were made. The images or other third party material in this article are included in the article's Creative Commons licence, unless indicated otherwise in a credit line to the material. If material is not included in the article's Creative Commons licence and your intended use is not permitted by statutory regulation or exceeds the permitted use, you will need to obtain permission directly from the copyright holder. To view a copy of this licence, visit http://creativecommons.org/licenses/by/4.0/. 
with $m, n \in \mathbb{N}_{0}:=\mathbb{N} \cup\{0\}$, and $\delta_{m, n}$ is the Kronecker delta [10, 13].

In 1975, Koornwinder [6] gave a general method to derive multivariate orthogonal polynomials from univariate orthogonal polynomials. One of the examples of Koornwinder's method is the Jacobi polynomials on the triangle

$$
\begin{aligned}
P_{n, k}^{(a, b, c)}(x, y) & =P_{n-k}^{(2 k+b+c+1, a)}(2 x-1)(1-x)^{k} P_{k}^{(c, b)}\left(\frac{2 y}{1-x}-1\right) \\
& =\widetilde{P}_{n-k}^{(2 k+b+c+1, a)}(x)(1-x)^{k} \widetilde{P}_{k}^{(c, b)}\left(\frac{y}{1-x}\right), \quad k=0,1, \ldots, n, n=0,1, \ldots,
\end{aligned}
$$

which are orthogonal on the two-dimensional simplex

$$
\mathbb{T}^{2}:=\left\{(x, y) \in \mathbb{R}^{2}: x, y>0,1-x-y>0\right\},
$$

with respect to the weight function $w_{a, b, c}(x, y)=x^{a} y^{b}(1-x-y)^{c}$ for $a, b, c>-1$,

$$
\begin{aligned}
\iint_{\mathbb{T}^{2}} P_{n, k}^{(a, b, c)}(x, y) P_{r, s}^{(a, b, c)}(x, y) x^{a} y^{b}(1-x-y)^{c} d x d y \\
=\frac{\Gamma(a+n-k+1) \Gamma(b+c+n+k+2) \Gamma(b+k+1) \Gamma(c+k+1)}{(n-k) ! k !(2 n+a+b+c+2)(2 k+b+c+1) \Gamma(n+k+a+b+c+2) \Gamma(k+b+c+1)} \\
\quad \times \delta_{n, r} \delta_{k, s} .
\end{aligned}
$$

In [9], Olver et al. introduced a bivariate four-parameter variant of the Koornwinder polynomials on the triangle defined by

$$
P_{n, k}^{(a, b, c, d)}(x, y)=\widetilde{P}_{n-k}^{(2 k+b+c+d+1, a)}(x)(1-x)^{k} \widetilde{P}_{k}^{(c, b)}\left(\frac{y}{1-x}\right)
$$

where $a, b, c, d>-1, n$ and $k$ are integers satisfying $0 \leq k \leq n$. These polynomials are orthogonal on the two-dimensional simplex $\mathbb{T}^{2}$, defined in (5), with respect to the weight function

$$
w_{a, b, c, d}(x, y)=x^{a} y^{b}(1-x-y)^{c}(1-x)^{d} .
$$

Indeed, it follows that

$$
\begin{aligned}
& \iint_{\mathbb{T}^{2}} P_{n, k}^{(a, b, c, d)}(x, y) P_{r, s}^{(a, b, c, d)}(x, y) x^{a} y^{b}(1-x-y)^{c}(1-x)^{d} d x d y \\
& =\frac{\Gamma(a+n-k+1)}{(n-k) ! k !} \\
& \quad \times \frac{\Gamma(b+c+d+n+k+2) \Gamma(b+k+1) \Gamma(c+k+1)}{(2 n+a+b+c+d+2)(2 k+b+c+1) \Gamma(n+k+a+b+c+d+2) \Gamma(k+b+c+1)} \\
& \quad \times \delta_{n, r} \delta_{k, s} .
\end{aligned}
$$

In the case $d=0$, (8) gives the bivariate Koornwinder polynomials on the triangle defined in (4). Olver et al. [9] obtained sparse recurrence relations by using some differential relations mapping $P_{n, k}^{(a, b, c, d)}(x, y)$ to scalar multiple of $P_{\widetilde{n}, \widetilde{k}}^{\widetilde{a}, \widetilde{c}, \widetilde{d})}(x, y)$, where the new parameters in $P_{\widetilde{n}, \widetilde{k}}^{(\widetilde{a}, \widetilde{c}, \widetilde{c}, \widetilde{d})}(x, y)$ are $n, k, a, b, c$, or $d$, respectively, or these values incremented or 
decremented by 1 . This approach allows one to derive new recurrence relations for the bivariate (classical) Koornwinder polynomials $P_{n, k}^{(a, b, c)}(x, y)$ (see [9]).

Let

$$
\mathbb{T}^{3}:=\left\{(x, y, z) \in \mathbb{R}^{3}: x, y, z>0,1-x-y-z>0\right\}
$$

be the three-dimensional simplex. In [3], Dunkl and Xu introduced the following family of three-variable polynomials:

$$
\begin{aligned}
P_{n}^{(\alpha, \beta, \gamma, \delta)}(x, y, z):= & P_{n_{1}, n_{2}, n_{3}}^{(\alpha, \beta, \gamma)}(x, y, z) \\
= & (1-x)^{n_{2}}(1-x-y)^{n_{3}} P_{n_{1}}^{\left(\beta+\gamma+\delta+2 n_{2}+2 n_{3}+2, \alpha\right)}(2 x-1) \\
& \times P_{n_{2}}^{\left(\gamma+\delta+2 n_{3}+1, \beta\right)}\left(\frac{2 y}{1-x}-1\right) P_{n_{3}}^{(\delta, \gamma)}\left(\frac{2 z}{1-x-y}-1\right),
\end{aligned}
$$

where $n_{1}, n_{2}, n_{3}, n \in \mathbb{N}_{0}$ and $n_{1}+n_{2}+n_{3}=n$. These polynomials are orthogonal on the simplex $\mathbb{T}^{3}$ with respect to the weight function

$$
w_{\alpha, \beta, \gamma, \delta}(x, y, z)=x^{\alpha} y^{\beta} z^{\gamma}(1-x-y-z)^{\delta}
$$

for $\alpha, \beta, \gamma, \delta>-1$ (see, for details, [3]).

In this paper, we consider a six-parameter variant of the three-variable polynomials (10) defined as

$$
\begin{aligned}
P_{n}^{(\alpha, \beta, \gamma, \delta, a, b)}(x, y, z):= & P_{n_{1}, n_{2}, n_{3}}^{(\alpha, \beta, \gamma, a, b)}(x, y, z) \\
= & (1-x)^{n_{2}}(1-x-y)^{n_{3}} P_{n_{1}}^{\left(\beta+\gamma+\delta+a+b+2 n_{2}+2 n_{3}+2, \alpha\right)}(2 x-1) \\
& \times P_{n_{2}}^{\left(\gamma+\delta+2 n_{3}+b+1, \beta\right)}\left(\frac{2 y}{1-x}-1\right) P_{n_{3}}^{(\delta, \gamma)}\left(\frac{2 z}{1-x-y}-1\right),
\end{aligned}
$$

where $n_{1}, n_{2}, n_{3}, n \in \mathbb{N}_{0}$ and $n_{1}+n_{2}+n_{3}=n$. The polynomials in (11) are orthogonal with respect to the weight function

$$
w_{\alpha, \beta, \gamma, \delta, a, b}(x, y, z)=x^{\alpha} y^{\beta} z^{\gamma}(1-x-y-z)^{\delta}(1-x)^{a}(1-x-y)^{b}
$$

for $\alpha, \beta, \gamma, \delta, a, b>-1$ on the simplex $\mathbb{T}^{3}$ defined in (9). More precisely,

$$
\begin{gathered}
\iiint_{\mathbb{T}^{3}} w_{\alpha, \beta, \gamma, \delta, a, b}(x, y, z) P_{n_{1}, n_{2}, n_{3}}^{(\alpha, \beta, \gamma, \delta, b)}(x, y, z) P_{m_{1}, m_{2}, m_{3}}^{(\alpha, \beta, \gamma, a, b)}(x, y, z) d x d y d z \\
=h_{n_{1}}^{\left(\beta+\gamma+\delta+a+b+2 n_{2}+2 n_{3}+2, \alpha\right)} h_{n_{2}}^{\left(\gamma+\delta+2 n_{3}+b+1, \beta\right)} h_{n_{3}}^{(\delta, \gamma)} \delta_{m_{1}, n_{1}} \delta_{m_{2}, n_{2}} \delta_{m_{3}, n_{3}},
\end{gathered}
$$

where $h_{n}^{(a, b)}$ is given in (3). In the case $a=b=0$, (11) gives the orthogonal polynomials given by (10) on the simplex $\mathbb{T}^{3}$ in [3].

By the motivation of the bivariate four-parameter variant of the Koornwinder polynomials (7) defined by Olver et al. and the six-parameter variant of the three-variable polynomials given by (11), we can define a $2 \mathrm{~d}$-parameter variant of the multivariate polynomials on the simplex. 
In general, let $\mathbf{x}_{j}$ and $\mathbf{n}^{j}$ be defined as

$$
\mathbf{x}_{0}=0, \quad \mathbf{x}_{j}=\left(x_{1}, \ldots, x_{j}\right), \quad 1 \leq j \leq d,
$$

and

$$
\mathbf{n}^{j}=\left(n_{j}, \ldots, n_{d}\right), \quad 1 \leq j \leq d .
$$

Also, let $\mathbf{x}=\left(x_{1}, \ldots, x_{d}\right), \mathbf{n}=\left(n_{1}, \ldots, n_{d}\right),|\mathbf{n}|=n_{1}+\cdots+n_{d}=n, \boldsymbol{\alpha}=\left(\alpha_{1}, \ldots, \alpha_{2 d}\right)$ and

$$
\begin{aligned}
& \widetilde{\boldsymbol{\alpha}}^{d+j+1}=\left(\alpha_{d+j+1}, \ldots, \alpha_{2 d}\right), \quad 1 \leq j \leq d-1, \\
& \boldsymbol{\alpha}^{j}=\left(\alpha_{j}, \ldots, \alpha_{d+1}\right), \quad 1 \leq j \leq d+1 .
\end{aligned}
$$

A $2 d$-parameter variant of the $d$-variable polynomials on the simplex

$$
\mathbb{T}^{d}:=\left\{\mathbf{x} \in \mathbb{R}^{d}: x_{1}, x_{2}, \ldots, x_{d}>0,1-x_{1}-x_{2}-\cdots-x_{d}>0\right\}
$$

is defined by

$$
P_{\mathbf{n}}^{(\alpha)}(\mathbf{x})=\prod_{j=1}^{d}\left(\frac{1-\left|\mathbf{x}_{j}\right|}{1-\left|\mathbf{x}_{j-1}\right|}\right)^{\left|\mathbf{n}^{j+1}\right|} P_{n_{j}}^{\left(a_{j}, b_{j}\right)}\left(\frac{2 x_{j}}{1-\left|\mathbf{x}_{j-1}\right|}-1\right),
$$

where $P_{n_{j}}^{\left(a_{j}, b_{j}\right)}\left(x_{j}\right)$ indicates the classical univariate Jacobi polynomial defined in (1), and $a_{j}=2\left|\mathbf{n}^{j+1}\right|+\left|\boldsymbol{\alpha}^{j+1}\right|+\left|\widetilde{\boldsymbol{\alpha}}^{d+j+1}\right|+d-j, b_{j}=\alpha_{j}$. These polynomials are orthogonal with respect to the weight function $W_{\alpha}(\mathbf{x})=x_{1}^{\alpha_{1}} \cdots x_{d}^{\alpha_{d}}\left(1-\left|x_{d}\right|\right)^{\alpha_{d+1}} \prod_{j=1}^{d-1}\left(1-\left|x_{j}\right|\right)^{\alpha_{d+j+1}}$ for $\alpha_{j}>-1, j=$ $1,2, \ldots, 2 d$ where $\left|\mathbf{x}_{j}\right|=x_{1}+\cdots+x_{j}$. In fact, it follows that

$$
\int_{T^{d}} W_{\alpha}(\mathbf{x}) P_{\mathbf{n}}^{(\boldsymbol{\alpha})}(\mathbf{x}) P_{\mathbf{m}}^{(\alpha)}(\mathbf{x}) \mathbf{d x}=h_{\mathbf{n}}^{(\boldsymbol{\alpha})} \delta_{\mathbf{n}, \mathbf{m}},
$$

where $\delta_{\mathbf{n}, \mathbf{m}}$ is Kronecker delta, $\mathbf{d x}=d x_{1} \cdots d x_{d}$ and

$$
\begin{aligned}
h_{\mathbf{n}}^{(\boldsymbol{\alpha})}= & \prod_{j=1}^{d} \frac{\Gamma\left(2\left|\mathbf{n}^{j+1}\right|+\left|\boldsymbol{\alpha}^{j+1}\right|+\left|\widetilde{\boldsymbol{\alpha}}^{d+j+1}\right|+d-j+1+n_{j}\right)}{n_{j} !\left(2\left|\mathbf{n}^{j+1}\right|+\left|\boldsymbol{\alpha}^{j+1}\right|+\left|\widetilde{\boldsymbol{\alpha}}^{d+j+1}\right|+d-j+1+\alpha_{j}+2 n_{j}\right)} \\
& \times \frac{\Gamma\left(1+\alpha_{j}+n_{j}\right)}{\Gamma\left(2\left|\mathbf{n}^{j+1}\right|+\left|\boldsymbol{\alpha}^{j+1}\right|+\left|\widetilde{\boldsymbol{\alpha}}^{d+j+1}\right|+d-j+1+\alpha_{j}+n_{j}\right)} .
\end{aligned}
$$

In the case $\alpha_{j}=0(j=d+2, \ldots, 2 d)$, (13) gives the classical orthogonal polynomials on the simplex $\mathbb{T}^{d}$ in [3].

In this paper, we apply the techniques used in [9] to the six-parameter variant of the three-variable polynomials on the simplex $\mathbb{T}^{3}$ rather than multivariate case, which is not easy to apply these techniques for the multivariate case. The relations obtained by these techniques give new results for the well-known three-variable polynomials with four parameters on the simplex $\mathbb{T}^{3}$. The structure of this paper is as follows. In the next section, we recall some differential relations and the corresponding sparse recurrence relations given in [9] for univariate (shifted) Jacobi polynomials $\widetilde{P}_{n}^{(a, b)}(x)$ and the bivariate polynomials 
$P_{n, k}^{(a, b, c, d)}(x, y)$. Some extra relations are also presented for the bivariate polynomials, which are new to the best of our knowledge. In Sect. 3, we give the corresponding differential relations for the polynomials (11) on the simplex $\mathbb{T}^{3}$. In Sect. 4, sparse recurrence relations for the polynomials (10) are deduced. Finally, in Sect. 5, some generating functions are obtained.

\section{Preliminaries}

In [9], Olver et al. obtained 24 differential relations and the corresponding sparse recurrence relations for a bivariate four-parameter variant of the Koornwinder polynomials defined by (7) on the triangle by using 12 differential relations and the corresponding sparse recurrence relations satisfied by univariate (shifted) Jacobi polynomials given by (2). In this paper, by using the techniques of reference [9] entirely, we present 36 sparse recurrence relations for a six-parameter variant of the three-variable polynomials. As a result of these sparse recurrence relations, we derive second order differential equations. For this purpose, we recall the results given in [9]. Let

$$
\begin{aligned}
& \widetilde{\mathcal{L}}_{1} u=\frac{d u}{d x}, \quad \widetilde{\mathcal{L}}_{1, a, b}^{+} u=(x a-(1-x) b) u-x(1-x) \frac{d u}{d x}, \\
& \widetilde{\mathcal{L}}_{2, a, b, n} u=(a+b+n+1) u+x \frac{d u}{d x}, \quad \widetilde{\mathcal{L}}_{2, a, n}^{+} u=(a+(1-x) n) u-x(1-x) \frac{d u}{d x}, \\
& \widetilde{\mathcal{L}}_{3, a, b, n} u=(a+b+n+1) u-(1-x) \frac{d u}{d x}, \quad \widetilde{\mathcal{L}}_{3, b, n}^{+} u=(b+x n) u+x(1-x) \frac{d u}{d x}, \\
& \widetilde{\mathcal{L}}_{4, a, b, n} u=(x a-(1-x)(b+n+1)) u-x(1-x) \frac{d u}{d x}, \quad \widetilde{\mathcal{L}}_{4, n}^{+} u=-n u+x \frac{d u}{d x}, \\
& \widetilde{\mathcal{L}}_{5, a, b, n} u=(x(a+n+1)-(1-x) b) u-x(1-x) \frac{d u}{d x}, \quad \widetilde{\mathcal{L}}_{5, n}^{+} u=n u+(1-x) \frac{d u}{d x}, \\
& \widetilde{\mathcal{L}}_{6, b} u=b u+x \frac{d u}{d x}, \quad \widetilde{\mathcal{L}}_{6, a}^{+} u=a u-(1-x) \frac{d u}{d x} .
\end{aligned}
$$

Then the following sparse recurrence relations for Jacobi polynomials hold true:

$$
\begin{array}{ll}
\widetilde{\mathcal{L}}_{1} \widetilde{P}_{n}^{(a, b)}(x)=(n+a+b+1) \widetilde{P}_{n-1}^{(a+1, b+1)}(x), & \widetilde{\mathcal{L}}_{1, a, b}^{+} \widetilde{P}_{n}^{(a, b)}(x)=(n+1) \widetilde{P}_{n+1}^{(a-1, b-1)}(x), \\
\widetilde{\mathcal{L}}_{2, a, b, n} \widetilde{P}_{n}^{(a, b)}(x)=(n+a+b+1) \widetilde{P}_{n}^{(a+1, b)}(x), & \widetilde{\mathcal{L}}_{2, a, n}^{+} \widetilde{P}_{n}^{(a, b)}(x)=(n+a) \widetilde{P}_{n}^{(a-1, b)}(x), \\
\widetilde{\mathcal{L}}_{3, a, b, n} \widetilde{P}_{n}^{(a, b)}(x)=(n+a+b+1) \widetilde{P}_{n}^{(a, b+1)}(x), & \widetilde{\mathcal{L}}_{3, b, n}^{+} \widetilde{P}_{n}^{(a, b)}(x)=(n+b) \widetilde{P}_{n}^{(a, b-1)}(x), \\
\widetilde{\mathcal{L}}_{4, a, b, n} \widetilde{P}_{n}^{(a, b)}(x)=(n+1) \widetilde{P}_{n+1}^{(a-1, b)}(x), & \widetilde{\mathcal{L}}_{4, n}^{+} \widetilde{P}_{n}^{(a, b)}(x)=(n+b) \widetilde{P}_{n-1}^{(a+1, b)}(x), \\
\widetilde{\mathcal{L}}_{5, a, b, n} \widetilde{P}_{n}^{(a, b)}(x)=(n+1) \widetilde{P}_{n+1}^{(a, b-1)}(x), & \widetilde{\mathcal{L}}_{5, n}^{+} \widetilde{P}_{n}^{(a, b)}(x)=(n+a) \widetilde{P}_{n-1}^{(a, b+1)}(x), \\
\widetilde{\mathcal{L}}_{6, b} \widetilde{P}_{n}^{(a, b)}(x)=(n+b) \widetilde{P}_{n}^{(a+1, b-1)}(x), & \widetilde{\mathcal{L}}_{6, a}^{+} \widetilde{P}_{n}^{(a, b)}(x)=(n+a) \widetilde{P}_{n}^{(a-1, b+1)}(x) .
\end{array}
$$

Moreover, the following second order differential relations are satisfied:

$$
\begin{aligned}
& \widetilde{\mathcal{L}}_{1, a, b}^{+} \widetilde{\mathcal{L}}_{1} \widetilde{P}_{n}^{(a-1, b-1)}(x)=n(n+a+b-1) \widetilde{P}_{n}^{(a-1, b-1)}(x), \\
& \widetilde{\mathcal{L}}_{1} \widetilde{\mathcal{L}}_{1, a, b}^{+} \widetilde{P}_{n}^{(a, b)}(x)=(n+1)(a+b+n) \widetilde{P}_{n}^{(a, b)}(x), \\
& \widetilde{\mathcal{L}}_{2, a, n}^{+} \widetilde{\mathcal{L}}_{2, a, b, n} \widetilde{P}_{n}^{(a-1, b+1)}(x)=(n+a)(n+a+b+1) \widetilde{P}_{n}^{(a-1, b+1)}(x), \\
& \widetilde{\mathcal{L}}_{2, a, b, n} \widetilde{\mathcal{L}}_{2, a, n}^{+} \widetilde{P}_{n}^{(a, b+1)}(x)=(n+a)(n+a+b+1) \widetilde{P}_{n}^{(a, b+1)}(x),
\end{aligned}
$$




$$
\begin{aligned}
& \widetilde{\mathcal{L}}_{3, b, n}^{+} \widetilde{\mathcal{L}}_{3, a, b, n} \widetilde{P}_{n}^{(a+1, b-1)}(x)=(n+b)(n+a+b+1) \widetilde{P}_{n}^{(a+1, b-1)}(x), \\
& \widetilde{\mathcal{L}}_{3, a, b, n} \widetilde{\mathcal{L}}_{3, b, n}^{+} \widetilde{P}_{n}^{(a+1, b)}(x)=(n+b)(n+a+b+1) \widetilde{P}_{n}^{(a+1, b)}(x), \\
& \widetilde{\mathcal{L}}_{4, n}^{+} \widetilde{\mathcal{L}}_{4, a, b, n} \widetilde{P}_{n-1}^{(a, b+1)}(x)=n(n+b+1) \widetilde{P}_{n-1}^{(a, b+1)}(x), \\
& \widetilde{\mathcal{L}}_{4, a, b, n} \widetilde{\mathcal{L}}_{4, n}^{+} \widetilde{P}_{n}^{(a-1, b+1)}(x)=n(n+b+1) \widetilde{P}_{n}^{(a-1, b+1)}(x), \\
& \widetilde{\mathcal{L}}_{5, n}^{+} \widetilde{\mathcal{L}}_{5, a, b, n} \widetilde{P}_{n-1}^{(a+1, b)}(x)=n(n+a+1) \widetilde{P}_{n-1}^{(a+1, b)}(x), \\
& \widetilde{\mathcal{L}}_{5, a, b, n} \widetilde{\mathcal{L}}_{5, n}^{+} \widetilde{P}_{n}^{(a+1, b-1)}(x)=n(n+a+1) \widetilde{P}_{n}^{(a+1, b-1)}(x), \\
& \widetilde{\mathcal{L}}_{6, a}^{+} \widetilde{\mathcal{L}}_{6, b} \widetilde{P}_{n}^{(a-1, b)}(x)=(n+a)(n+b) \widetilde{P}_{n}^{(a-1, b)}(x), \\
& \widetilde{\mathcal{L}}_{6, b} \widetilde{\mathcal{L}}_{6, a}^{+} \widetilde{P}_{n}^{(a, b-1)}(x)=(n+a)(n+b) \widetilde{P}_{n}^{(a, b-1)}(x) .
\end{aligned}
$$

From the above relations we also have the following second order differential equations for the univariate shifted Jacobi polynomials:

$$
\begin{aligned}
& \widetilde{\mathcal{L}}_{1, a+1, b+1}^{+} \widetilde{\mathcal{L}}_{1} \widetilde{P}_{n}^{(a, b)}(x)=n(n+a+b+1) \widetilde{P}_{n}^{(a, b)}(x), \\
& \widetilde{\mathcal{L}}_{1} \widetilde{\mathcal{L}}_{1, a, b}^{+} \widetilde{P}_{n}^{(a, b)}(x)=(n+1)(a+b+n) \widetilde{P}_{n}^{(a, b)}(x), \\
& \widetilde{\mathcal{L}}_{2, a+1, n}^{+} \widetilde{\mathcal{L}}_{2, a+1, b-1, n} \widetilde{P}_{n}^{(a, b)}(x)=(n+a+1)(n+a+b+1) \widetilde{P}_{n}^{(a, b)}(x), \\
& \widetilde{\mathcal{L}}_{2, a, b-1, n} \widetilde{\mathcal{L}}_{2, a, n}^{+} \widetilde{P}_{n}^{(a, b)}(x)=(n+a)(n+a+b) \widetilde{P}_{n}^{(a, b)}(x), \\
& \widetilde{\mathcal{L}}_{3, b+1, n}^{+} \widetilde{\mathcal{L}}_{3, a-1, b+1, n} \widetilde{P}_{n}^{(a, b)}(x)=(n+b+1)(n+a+b+1) \widetilde{P}_{n}^{(a, b)}(x), \\
& \widetilde{\mathcal{L}}_{3, a-1, b, n} \widetilde{\mathcal{L}}_{3, b, n}^{+} \widetilde{P}_{n}^{(a, b)}(x)=(n+b)(n+a+b) \widetilde{P}_{n}^{(a, b)}(x), \\
& \widetilde{\mathcal{L}}_{4, n+1}^{+} \widetilde{\mathcal{L}}_{4, a, b-1, n+1} \widetilde{P}_{n}^{(a, b)}(x)=(n+1)(n+b+1) \widetilde{P}_{n}^{(a, b)}(x), \\
& \widetilde{\mathcal{L}}_{4, a+1, b-1, n} \widetilde{\mathcal{L}}_{4, n}^{+} \widetilde{P}_{n}^{(a, b)}(x)=n(n+b) \widetilde{P}_{n}^{(a, b)}(x), \\
& \widetilde{\mathcal{L}}_{5, n+1}^{+} \widetilde{\mathcal{L}}_{5, a-1, b, n+1} \widetilde{P}_{n}^{(a, b)}(x)=(n+1)(n+a+1) \widetilde{P}_{n}^{(a, b)}(x), \\
& \widetilde{\mathcal{L}}_{5, a-1, b+1, n} \widetilde{\mathcal{L}}_{5, n}^{+} \widetilde{P}_{n}^{(a, b)}(x)=n(n+a) \widetilde{P}_{n}^{(a, b)}(x), \\
& \widetilde{\mathcal{L}}_{6, a+1}^{+} \widetilde{\mathcal{L}}_{6, b} \widetilde{P}_{n}^{(a, b)}(x)=(n+a+1)(n+b) \widetilde{P}_{n}^{(a, b)}(x), \\
& \widetilde{\mathcal{L}}_{6, b+1} \widetilde{\mathcal{L}}_{6, a}^{+} \widetilde{P}_{n}^{(a, b)}(x)=(n+a)(n+b+1) \widetilde{P}_{n}^{(a, b)}(x) .
\end{aligned}
$$

The first, second and the last two relations give exactly the second order differential equation for the univariate shifted Jacobi polynomials. Moreover, the third, fourth, seventh, and eighth relations give the second order differential equation for the univariate shifted Jacobi polynomials multiplied by $x$, while the fifth, sixth, ninth, and tenth relations give the second order differential equation for the univariate shifted Jacobi polynomials multiplied by $1-x$.

By using these 12 relations, in [9] 24 relations for the bivariate orthogonal polynomials $P_{n, k}^{(a, b, c, d)}(x, y)$ defined in (7) are derived. These 24 relations map $P_{n, k}^{(a, b, c, d)}(x, y)$ to a scalar multiple of $P_{\widetilde{n}, \widetilde{k}}^{(\widetilde{a}, \widetilde{c}, \widetilde{d})}(x, y)$, where the new parameters in $P_{\widetilde{n}, \widetilde{k}}^{(\widetilde{a}, \widetilde{b}, \widetilde{d})}(x, y)$ are $n, k, a, b, c$ or $d$, respectively, incremented or decremented by 0 or 1 . Following the notations in [9], let

$$
\mathcal{M}_{0,1} u=\frac{\partial u}{\partial y}
$$




$$
\begin{aligned}
& \mathcal{M}_{0,2} u=(k+b+c+1) u+y \frac{\partial u}{\partial y}, \\
& \mathcal{M}_{0,3} u=(k+b+c+1) u-(1-x-y) \frac{\partial u}{\partial y}, \\
& \mathcal{M}_{0,4} u=(y c-(1-x-y)(b+k+1)) u-y(1-x-y) \frac{\partial u}{\partial y}, \\
& \mathcal{M}_{0,5} u=(y(c+k+1)-(1-x-y) b) u-y(1-x-y) \frac{\partial u}{\partial y}, \\
& \mathcal{M}_{0,6} u=b u+y \frac{\partial u}{\partial y} \\
& \mathcal{M}_{0,1}^{+} u=(y c-(1-x-y) b) u-y(1-x-y) \frac{\partial u}{\partial y}, \\
& \mathcal{M}_{0,2}^{+} u=\left(c+k-\frac{y k}{1-x}\right) u-\frac{y(1-x-y)}{1-x} \frac{\partial u}{\partial y}, \\
& \mathcal{M}_{0,3}^{+} u=\left(b+\frac{k y}{1-x}\right) u+\frac{y(1-x-y)}{1-x} \frac{\partial u}{\partial y}, \\
& \mathcal{M}_{0,4}^{+} u=-\frac{k}{1-x} u+\frac{y}{1-x} \frac{\partial u}{\partial y}, \\
& \mathcal{M}_{0,5}^{+} u=\frac{k}{1-x} u+\left(1-\frac{y}{1-x}\right) \frac{\partial u}{\partial y}, \\
& \mathcal{M}_{0,6}^{+} u=c u-(1-x-y) \frac{\partial u}{\partial y},
\end{aligned}
$$

and

$$
\begin{aligned}
& \mathcal{M}_{1,0} u=\frac{k u}{1-x}+\frac{\partial u}{\partial x}-\frac{y}{1-x} \frac{\partial u}{\partial y}, \\
& \mathcal{M}_{1,0}^{+} u=(x(k+a+b+c+d+1)-a) u-x(1-x) \frac{\partial u}{\partial x}+x y \frac{\partial u}{\partial y}, \\
& \mathcal{M}_{2,0} u=(n+k+a+b+c+d+2) u+\frac{k x}{1-x} u+x \frac{\partial u}{\partial x}-\frac{x y}{1-x} \frac{\partial u}{\partial y}, \\
& \mathcal{M}_{2,0}^{+} u=(n+k+b+c+d+1-x n) u-x(1-x) \frac{\partial u}{\partial x}+x y \frac{\partial u}{\partial y} \\
& \mathcal{M}_{3,0} u=(n+a+b+c+d+2) u-(1-x) \frac{\partial u}{\partial x}+y \frac{\partial u}{\partial y}, \\
& \mathcal{M}_{3,0}^{+} u=(a+x n) u+x(1-x) \frac{\partial u}{\partial x}-x y \frac{\partial u}{\partial y}, \\
& \mathcal{M}_{4,0} u=(x(n+a+b+c+d+2)-a-n+k-1) u-x(1-x) \frac{\partial u}{\partial x}+x y \frac{\partial u}{\partial y} \\
& \mathcal{M}_{4,0}^{+} u=\frac{k u}{1-x}-n u+x \frac{\partial u}{\partial x}-\frac{x y}{1-x} \frac{\partial u}{\partial y}, \\
& \mathcal{M}_{5,0} u=x(n+a+b+c+d+2) u-a u-x(1-x) \frac{\partial u}{\partial x}+x y \frac{\partial u}{\partial y}, \\
& \mathcal{M}_{5,0}^{+} u=n u+(1-x) \frac{\partial u}{\partial x}-y \frac{\partial u}{\partial y},
\end{aligned}
$$




$$
\begin{aligned}
& \mathcal{M}_{6,0} u=a u+\frac{k x}{1-x} u+x \frac{\partial u}{\partial x}-\frac{x y}{1-x} \frac{\partial u}{\partial y}, \\
& \mathcal{M}_{6,0}^{+} u=(k+b+c+d+1) u-(1-x) \frac{\partial u}{\partial x}+y \frac{\partial u}{\partial y} .
\end{aligned}
$$

The sparse recurrence relations are given as follows:

$$
\begin{aligned}
& \mathcal{M}_{0,1} P_{n, k}^{(a, b, c, d)}(x, y)=(k+b+c+1) P_{n-1, k-1}^{(a, b+1, c+1, d)}(x, y), \\
& \mathcal{M}_{0,1}^{+} P_{n, k}^{(a, b, c, d)}(x, y)=(k+1) P_{n+1, k+1}^{(a, b-1, c-1, d)}(x, y), \\
& \mathcal{M}_{0,2} P_{n, k}^{(a, b, c, d)}(x, y)=(k+b+c+1) P_{n, k}^{(a, b, c+1, d-1)}(x, y), \\
& \mathcal{M}_{0,2}^{+} P_{n, k}^{(a, b, c, d)}(x, y)=(k+c) P_{n, k}^{(a, b, c-1, d+1)}(x, y), \\
& \mathcal{M}_{0,3} P_{n, k}^{(a, b, c, d)}(x, y)=(k+b+c+1) P_{n, k}^{(a, b+1, c, d-1)}(x, y), \\
& \mathcal{M}_{0,3}^{+} P_{n, k}^{(a, b, c, d)}(x, y)=(k+b) P_{n, k}^{(a, b-1, c, d+1)}(x, y), \\
& \mathcal{M}_{0,4} P_{n, k}^{(a, b, c, d)}(x, y)=(k+1) P_{n+1, k+1}^{(a, b, c-1, d-1)}(x, y), \\
& \mathcal{M}_{0,4}^{+} P_{n, k}^{(a, b, c, d)}(x, y)=(k+b) P_{n-1, k-1}^{(a, b, c+1, d+1)}(x, y), \\
& \mathcal{M}_{0,5} P_{n, k}^{(a, b, c, d)}(x, y)=(k+1) P_{n+1, k+1}^{(a, b-1, c, d-1)}(x, y), \\
& \mathcal{M}_{0,5}^{+} P_{n, k}^{(a, b, c, d)}(x, y)=(k+c) P_{n-1, k-1}^{(a, b+1, c, d+1)}(x, y), \\
& \mathcal{M}_{0,6} P_{n, k}^{(a, b, c, d)}(x, y)=(k+b) P_{n, k}^{(a, b-1, c+1, d)}(x, y), \\
& \mathcal{M}_{0,6}^{+} P_{n, k}^{(a, b, c, d)}(x, y)=(k+c) P_{n, k}^{(a, b+1, c-1, d)}(x, y),
\end{aligned}
$$

and

$$
\begin{aligned}
& \mathcal{M}_{1,0} P_{n, k}^{(a, b, c, d)}(x, y)=(n+k+a+b+c+d+2) P_{n-1, k}^{(a+1, b, c, d+1)}(x, y), \\
& \mathcal{M}_{1,0}^{+} P_{n, k}^{(a, b, c, d)}(x, y)=(n-k+1) P_{n+1, k}^{(a-1, b, c, d-1)}(x, y), \\
& \mathcal{M}_{2,0} P_{n, k}^{(a, b, c, d)}(x, y)=(n+k+a+b+c+d+2) P_{n, k}^{(a, b, c, d+1)}(x, y), \\
& \mathcal{M}_{2,0}^{+} P_{n, k}^{(a, b, c, d)}(x, y)=(n+k+b+c+d+1) P_{n, k}^{(a, b, c, d-1)}(x, y), \\
& \mathcal{M}_{3,0} P_{n, k}^{(a, b, c, d)}(x, y)=(n+k+a+b+c+d+2) P_{n, k}^{(a+1, b, c, d)}(x, y), \\
& \mathcal{M}_{3,0}^{+} P_{n, k}^{(a, b, c, d)}(x, y)=(n-k+a) P_{n, k}^{(a-1, b, c, d)}(x, y), \\
& \mathcal{M}_{4,0} P_{n, k}^{(a, b, c, d)}(x, y)=(n-k+1) P_{n+1, k}^{(a, b, c, d-1)}(x, y), \\
& \mathcal{M}_{4,0}^{+} P_{n, k}^{(a, b, c, d)}(x, y)=(n-k+a) P_{n-1, k}^{(a, b, c, d+1)}(x, y), \\
& \mathcal{M}_{5,0} P_{n, k}^{(a, b, c, d)}(x, y)=(n-k+1) P_{n+1, k}^{(a-1, b, c, d)}(x, y), \\
& \mathcal{M}_{5,0}^{+} P_{n, k}^{(a, b, c, d)}(x, y)=(n+k+b+c+d+1) P_{n-1, k}^{(a+1, b, c, d)}(x, y), \\
& \mathcal{M}_{6,0} P_{n, k}^{(a, b, c, d)}(x, y)=(n-k+a) P_{n, k}^{(a-1, b, c, d+1)}(x, y), \\
& \mathcal{M}_{6,0}^{+} P_{n, k}^{(a, b, c, d)}(x, y)=(n+k+b+c+d+1) P_{n, k}^{(a+1, b, c, d-1)}(x, y) .
\end{aligned}
$$

Moreover, the following second order differential relations hold:

$$
\mathcal{M}_{0,1}^{+} \mathcal{M}_{0,1} P_{n, k}^{(a, b-1, c-1, d)}(x, y)=k(k+b+c-1) P_{n, k}^{(a, b-1, c-1, d)}(x, y),
$$




$$
\begin{aligned}
& \mathcal{M}_{0,1} \mathcal{M}_{0,1}^{+} P_{n, k}^{(a, b, c, d)}(x, y)=(k+1)(k+b+c) P_{n, k}^{(a, b, c, d)}(x, y), \\
& \mathcal{M}_{0,2}^{+} \mathcal{M}_{0,2} P_{n, k}^{(a, b+1, c-1, d)}(x, y)=(k+c)(k+b+c+1) P_{n, k}^{(a, b+1, c-1, d)}(x, y), \\
& \mathcal{M}_{0,2} \mathcal{M}_{0,2}^{+} P_{n, k}^{(a, b+1, c, d)}(x, y)=(k+c)(k+b+c+1) P_{n, k}^{(a, b+1, c, d)}(x, y), \\
& \mathcal{M}_{0,3}^{+} \mathcal{M}_{0,3} P_{n, k}^{(a, b-1, c+1, d)}(x, y)=(k+b)(k+b+c+1) P_{n, k}^{(a, b-1, c+1, d)}(x, y), \\
& \mathcal{M}_{0,3} \mathcal{M}_{0,3}^{+} P_{n, k}^{(a, b, c+1, d)}(x, y)=(k+b)(k+b+c+1) P_{n, k}^{(a, b, c+1, d)}(x, y), \\
& \mathcal{M}_{0,4}^{+} \mathcal{M}_{0,4} P_{n, k-1}^{(a, b+1, c, d)}(x, y)=k(k+b+1) P_{n, k-1}^{(a, b+1, c, d)}(x, y), \\
& \mathcal{M}_{0,4} \mathcal{M}_{0,4}^{+} P_{n, k}^{(a, b+1, c-1, d)}(x, y)=k(k+b+1) P_{n, k}^{(a, b+1, c-1, d)}(x, y), \\
& \mathcal{M}_{0,5}^{+} \mathcal{M}_{0,5} P_{n, k-1}^{(a, b, c+1, d)}(x, y)=k(k+c+1) P_{n, k-1}^{(a, b, c+1, d)}(x, y), \\
& \mathcal{M}_{0,5} \mathcal{M}_{0,5}^{+} P_{n, k}^{(a, b-1, c+1, d)}(x, y)=k(k+c+1) P_{n, k}^{(a, b-1, c+1, d)}(x, y), \\
& \mathcal{M}_{0,6}^{+} \mathcal{M}_{0,6} P_{n, k}^{(a, b, c-1, d)}(x, y)=(k+b)(k+c) P_{n, k}^{(a, b, c-1, d)}(x, y), \\
& \mathcal{M}_{0,6} \mathcal{M}_{0,6}^{+} P_{n, k}^{(a, b-1, c, d)}(x, y)=(k+b)(k+c) P_{n, k}^{(a, b-1, c, d)}(x, y),
\end{aligned}
$$

and

$$
\begin{aligned}
& \mathcal{M}_{1,0}^{+} \mathcal{M}_{1,0} P_{n, k}^{(a-1, b, c, d-1)}(x, y)=(n-k)(n+k+a+b+c+d) P_{n, k}^{(a-1, b, c, d-1)}(x, y), \\
& \mathcal{M}_{1,0} \mathcal{M}_{1,0}^{+} P_{n, k}^{(a, b, c, d)}(x, y)=(n-k+1)(n+k+a+b+c+d+1) P_{n, k}^{(a, b, c, d)}(x, y), \\
& \mathcal{M}_{2,0}^{+} \mathcal{M}_{2,0} P_{n, k}^{(a+1, b, c, d-1)}(x, y)=(n+k+a+b+c+d+2) \\
& \times(n+k+b+c+d+1) P_{n, k}^{(a+1, b, c, d-1)}(x, y), \\
& \mathcal{M}_{2,0} \mathcal{M}_{2,0}^{+} P_{n, k}^{(a+1, b-1, c, d+1)}(x, y)=(n+k+a+b+c+d+2) \\
& \times(n+k+b+c+d+1) P_{n, k}^{(a+1, b-1, c, d+1)}(x, y), \\
& \mathcal{M}_{3,0}^{+} \mathcal{M}_{3,0} P_{n, k}^{(a-1, b+1, c, d)}(x, y)=(n+k+a+b+c+d+2)(n-k+a) P_{n, k}^{(a-1, b+1, c, d)}(x, y), \\
& \mathcal{M}_{3,0} \mathcal{M}_{3,0}^{+} P_{n, k}^{(a, b+1, c, d)}(x, y)=(n+k+a+b+c+d+2)(n-k+a) P_{n, k}^{(a, b+1, c, d)}(x, y), \\
& \mathcal{M}_{4,0}^{+} \mathcal{M}_{4,0} P_{n-1, k}^{(a+1, b, c, d)}(x, y)=(n-k)(n-k+a+1) P_{n-1, k}^{(a+1, b, c, d)}(x, y), \\
& \mathcal{M}_{4,0} \mathcal{M}_{4,0}^{+} P_{n, k}^{(a+1, b-1, c, d)}(x, y)=(n-k)(n-k+a+1) P_{n, k}^{(a+1, b-1, c, d)}(x, y), \\
& \mathcal{M}_{5,0}^{+} \mathcal{M}_{5,0} P_{n-1, k}^{(a, b+1, c, d)}(x, y)=(n-k)(n+k+b+c+d+2) P_{n-1, k}^{(a, b+1, c, d)}(x, y), \\
& \mathcal{M}_{5,0} \mathcal{M}_{5,0}^{+} P_{n, k}^{(a-1, b+1, c, d)}(x, y)=(n-k)(n+k+b+c+d+2) P_{n, k}^{(a-1, b+1, c, d)}(x, y), \\
& \mathcal{M}_{6,0}^{+} \mathcal{M}_{6,0} P_{n, k}^{(a, b, c, d-1)}(x, y)=(n-k+a)(n+k+b+c+d+1) P_{n, k}^{(a, b, c, d-1)}(x, y), \\
& \mathcal{M}_{6,0} \mathcal{M}_{6,0}^{+} P_{n, k}^{(a-1, b, c, d)}(x, y)=(n-k+a)(n+k+b+c+d+1) P_{n, k}^{(a-1, b, c, d)}(x, y) .
\end{aligned}
$$

The polynomials $P_{n, k}^{(a, b, c, d)}(x, y)$ defined in (7) satisfy the differential equations

$$
\begin{aligned}
L_{1} u:= & y(1-x-y) \frac{\partial^{2} u}{\partial y^{2}}+((b+1)(1-x)-(b+c+2) y) \frac{\partial u}{\partial y} \\
& +k(k+b+c+1) u \\
= & 0
\end{aligned}
$$


and

$$
\begin{aligned}
L_{2} u:= & x(1-x) \frac{\partial^{2} u}{\partial x^{2}}-2 x y \frac{\partial^{2} u}{\partial x \partial y}+y(1-y) \frac{\partial^{2} u}{\partial y^{2}}+(a+1-(a+b+c+d+3) x) \frac{\partial u}{\partial x} \\
& +\left(b+1-(a+b+c+d+3) y+\frac{y d}{1-x}\right) \frac{\partial u}{\partial y} \\
& +\left(n(n+a+b+c+d+2)-\frac{k d}{1-x}\right) u \\
= & 0 .
\end{aligned}
$$

Equation (15) is given in [9]. From $L_{2} u-\frac{1}{1-x} L_{1} u=0$, it also leads to

$$
\begin{aligned}
x(1-x) & \frac{\partial^{2} u}{\partial x^{2}}-2 x y \frac{\partial^{2} u}{\partial x \partial y}+\frac{x y^{2}}{1-x} \frac{\partial^{2} u}{\partial y^{2}}+(a+1-(a+b+c+d+3) x) \frac{\partial u}{\partial x} \\
& -\left(\frac{y(a+1-x(a+b+c+d+3))}{1-x}\right) \frac{\partial u}{\partial y} \\
& +\left(n(n+a+b+c+d+2)-\frac{k(k+b+c+d+1)}{1-x}\right) u \\
= & 0 .
\end{aligned}
$$

Remark 1 For second order differential equations in the first set given above, the first, second and the last two equations give exactly the second order equation in the form of (15). The third, fourth, seventh and eighth equations give Eq. (15) multiplied by $y$. The fifth, sixth, ninth and tenth equations give Eq. (15) multiplied by $1-x-y$.

For second order differential equations in the second set given above, the first, second and the last two equations give exactly the second order equation in the form of (17). The third, fourth, seventh and eighth equations give Eq. (17) multiplied by $x$. The fifth, sixth, ninth and tenth equations give Eq. (17) multiplied by $1-x$.

Remark 2 The partial differential equation (17) has also a monic solution [1], given by

$$
\begin{aligned}
\tilde{P}_{n, k}^{(a, b, c, d)}(x, y)= & (-1)^{n+k} \frac{\Gamma(a+n-k+1) \Gamma(a+b+c+d+n+k+2)}{\Gamma(a+1) \Gamma(a+b+c+d+2 n+2)} \\
& \times y^{k}{ }_{2} F_{1}\left(\begin{array}{c}
-n+k, a+b+c+d+n+k+2 \\
a+1
\end{array} \mid x\right),
\end{aligned}
$$

which can be also written as

$$
\tilde{P}_{n, k}^{(a, b, c, d)}(x, y)=\frac{(n-k) ! \Gamma(a+b+c+d+n+k+2)}{\Gamma(a+b+c+d+2 n+2)} y^{k} \widetilde{P}_{n-k}^{(b+c+d+2 k+1, a)}(x)
$$

in terms of the shifted Jacobi polynomials $\widetilde{P}_{n}^{(a, b)}(x)$ defined in (2). Notice that in this representation of the monic solution of the partial differential equation (17) the parameters of the shifted Jacobi polynomials depend on the degree i.e. they are varying orthogonal polynomials [14]. 


\section{Differential relations for $P_{n}^{(\alpha, \beta, \gamma, \delta, a, b)}(x, y, z)$}

By using the 12 differential relations given in Sect. 2 for the univariate shifted Jacobi polynomials $\widetilde{P}_{n}^{(a, b)}(x)$ defined in (2) and the 24 differential relations also given in Sect. 2 for the polynomials $P_{n, k}^{(a, b, c, d)}(x, y)$ defined in (7), we obtain 36 differential relations for $P_{n_{1}, n_{2}, n_{3}}^{(\alpha, \beta, \delta, a, j)}(x, y, z)$ defined in (11), which map $P_{n_{1}, n_{2}, n_{3}}^{(\alpha, \beta, \gamma, \alpha, b)}(x, y, z)$ to a scalar multiple of $P_{\widetilde{n}_{1}, \widetilde{n}_{2}, \widetilde{n}_{3}}^{(\widetilde{\alpha}, \widetilde{a}, \widetilde{b})}(x, y, z)$ where the parameters in the new family $P_{\widetilde{n_{1}}, \widetilde{n}_{2}, \widetilde{n}_{3}}^{(\widetilde{\alpha}, \widetilde{a}, \widetilde{a}, \widetilde{b})}(x, y, z)$ are $n_{1}, n_{2}, n_{3}$, $\alpha, \beta, \gamma, \delta, a, b$ incremented or decremented by 0 or 1 .

Let

$$
\begin{aligned}
& \mathcal{N}_{0,1} u=\frac{n_{3} u}{1-x-y}+\frac{\partial u}{\partial y}-\frac{z}{1-x-y} \frac{\partial u}{\partial z}, \\
& \mathcal{N}_{0,1}^{+} u=\left(y\left(\gamma+\delta+n_{3}+b+1\right)-(1-x-y) \beta\right) u-y(1-x-y) \frac{\partial u}{\partial y}+y z \frac{\partial u}{\partial z}, \\
& \mathcal{N}_{0,2} u=\left(n_{2}+2 n_{3}+\beta+\gamma+\delta+b+2\right) u+\frac{n_{3} y}{1-x-y} u+y \frac{\partial u}{\partial y}-\frac{y z}{1-x-y} \frac{\partial u}{\partial z}, \\
& \mathcal{N}_{0,2}^{+} u=\left(n_{2}+2 n_{3}+\gamma+\delta+b+1\right) u-\frac{\left(n_{2}+n_{3}\right) y}{1-x} u-\frac{y(1-x-y)}{1-x} \frac{\partial u}{\partial y}+\frac{y z}{1-x} \frac{\partial u}{\partial z}, \\
& \mathcal{N}_{0,3} u=\left(n_{2}+n_{3}+\beta+\gamma+\delta+b+2\right) u-(1-x-y) \frac{\partial u}{\partial y}+z \frac{\partial u}{\partial z}, \\
& \mathcal{N}_{0,3}^{+} u=\left(\beta+\frac{n_{2}+n_{3}}{1-x} y\right) u+\frac{y(1-x-y)}{1-x} \frac{\partial u}{\partial y}-\frac{y z}{1-x} \frac{\partial u}{\partial z}, \\
& \mathcal{N}_{0,4} u=\left(y\left(n_{3}+\gamma+\delta+b+1\right)-(1-x-y)\left(\beta+n_{2}+1\right)\right) u-y(1-x-y) \frac{\partial u}{\partial y}+y z \frac{\partial u}{\partial z}, \\
& \mathcal{N}_{0,4}^{+} u=-\frac{n_{2} u}{1-x}+\frac{n_{3} y}{(1-x)(1-x-y)} u+\frac{y}{1-x} \frac{\partial u}{\partial y}-\frac{\partial z}{(1-x)(1-x-y)} \frac{\partial u}{\partial z}, \\
& \mathcal{N}_{0,5} u=\left(y\left(n_{2}+n_{3}+\gamma+\delta+b+2\right)-(1-x-y) \beta\right) u-y(1-x-y) \frac{\partial u}{\partial y}+y z \frac{\partial u}{\partial z}, \\
& \mathcal{N}_{0,5}^{+} u=\frac{n_{2}+n_{3}}{1-x} u+\left(1-\frac{y}{1-x}\right) \frac{\partial u}{\partial y}-\frac{z}{1-x} \frac{\partial u}{\partial z}, \\
& \mathcal{N}_{0,6} u=\beta u+\frac{n_{3} y}{1-x-y} u+y \frac{\partial u}{\partial y}-\frac{y z}{1-x-y} \frac{\partial u}{\partial z}, \\
& \mathcal{N}_{0,6}^{+} u=\left(\gamma+\delta+n_{3}+b+1\right) u-(1-x-y) \frac{\partial u}{\partial y}+z \frac{\partial u}{\partial z},
\end{aligned}
$$

and

$$
\begin{aligned}
& \mathcal{N}_{1,0} u=\frac{n_{2}+n_{3}}{1-x} u+\frac{\partial u}{\partial x}-\frac{y}{1-x} \frac{\partial u}{\partial y}-\frac{z}{1-x} \frac{\partial u}{\partial z} \\
& \mathcal{N}_{1,0}^{+} u=\left(x\left(n_{2}+n_{3}+e+2\right)-\alpha\right) u-x(1-x) \frac{\partial u}{\partial x}+x y \frac{\partial u}{\partial y}+x z \frac{\partial u}{\partial z}, \\
& \mathcal{N}_{2,0} u=\left(n+n_{2}+n_{3}+e+3\right) u+\frac{\left(n_{2}+n_{3}\right) x u}{1-x}+x \frac{\partial u}{\partial x}-\frac{x y}{1-x} \frac{\partial u}{\partial y}-\frac{x z}{1-x} \frac{\partial u}{\partial z}, \\
& \mathcal{N}_{2,0}^{+} u=\left(n+n_{2}+n_{3}+e-\alpha+2-n x\right) u-x(1-x) \frac{\partial u}{\partial x}+x y \frac{\partial u}{\partial y}+x z \frac{\partial u}{\partial z}, \\
& \mathcal{N}_{3,0} u=(n+e+3) u-(1-x) \frac{\partial u}{\partial x}+y \frac{\partial u}{\partial y}+z \frac{\partial u}{\partial z},
\end{aligned}
$$




$$
\begin{aligned}
& \mathcal{N}_{3,0}^{+} u=(\alpha+n x) u+x(1-x) \frac{\partial u}{\partial x}-x y \frac{\partial u}{\partial y}-x z \frac{\partial u}{\partial z}, \\
& \mathcal{N}_{4,0} u=\left(x(n+e+3)-\alpha-n_{1}-1\right) u-x(1-x) \frac{\partial u}{\partial x}+x y \frac{\partial u}{\partial y}+x z \frac{\partial u}{\partial z}, \\
& \mathcal{N}_{4,0}^{+} u=-n u+\frac{\left(n_{2}+n_{3}\right) u}{1-x}+x \frac{\partial u}{\partial x}-\frac{x y}{1-x} \frac{\partial u}{\partial y}-\frac{x z}{1-x} \frac{\partial u}{\partial z} \\
& \mathcal{N}_{5,0} u=((n+e+3) x-\alpha) u-x(1-x) \frac{\partial u}{\partial x}+x y \frac{\partial u}{\partial y}+x z \frac{\partial u}{\partial z} \\
& \mathcal{N}_{5,0}^{+} u=n u+(1-x) \frac{\partial u}{\partial x}-y \frac{\partial u}{\partial y}-z \frac{\partial u}{\partial z}, \\
& \mathcal{N}_{6,0} u=\alpha u+\frac{\left(n_{2}+n_{3}\right) x u}{1-x}+x \frac{\partial u}{\partial x}-\frac{x y}{1-x} \frac{\partial u}{\partial y}-\frac{x z}{1-x} \frac{\partial u}{\partial z} \\
& \mathcal{N}_{6,0}^{+} u=\left(n_{2}+n_{3}+e-\alpha+2\right) u-(1-x) \frac{\partial u}{\partial x}+y \frac{\partial u}{\partial y}+z \frac{\partial u}{\partial z}
\end{aligned}
$$

and

$$
\begin{aligned}
& \mathcal{O}_{1,0} u=\frac{\partial u}{\partial z}, \\
& \mathcal{O}_{1,0}^{+} u=(z \delta-w \gamma) u-z w \frac{\partial u}{\partial z} \\
& \mathcal{O}_{2,0} u=\left(\delta+\gamma+n_{3}+1\right) u+z \frac{\partial u}{\partial z} \\
& \mathcal{O}_{2,0}^{+} u=\left(\delta+\frac{n_{3} w}{1-x-y}\right) u-\frac{z w}{1-x-y} \frac{\partial u}{\partial z} \\
& \mathcal{O}_{3,0} u=\left(\delta+\gamma+n_{3}+1\right) u-w \frac{\partial u}{\partial z}, \\
& \mathcal{O}_{3,0}^{+} u=\left(\gamma+\frac{n_{3} z}{1-x-y}\right) u+\frac{z w}{1-x-y} \frac{\partial u}{\partial z} \\
& \mathcal{O}_{4,0} u=\left(z \delta-w\left(\gamma+n_{3}+1\right)\right) u-z w \frac{\partial u}{\partial z} \\
& \mathcal{O}_{4,0}^{+} u=-\frac{n_{3} u}{1-x-y}+\frac{\partial u}{1-x-y} \frac{\partial z}{\partial z} \\
& \mathcal{O}_{6,0}^{+} u=\delta u-w \frac{\partial u}{\partial z}, \\
& \mathcal{O}_{5,0}^{+} u=\left(\left(\delta+n_{3}+1\right) z-w \gamma\right) u-z w \frac{\partial u}{\partial z} \\
& \mathcal{O}_{5,0} u
\end{aligned}
$$

where $n_{1}+n_{2}+n_{3}=n, \alpha+\beta+\gamma+\delta+a+b=e$ and $w=1-x-y-z$.

The differential relations above are chosen so that the recurrence relations in the next theorem are obtained for $\mathcal{N}_{i, 0}\left(\right.$ or $\left.\mathcal{N}_{i, 0}^{+}\right)$and $\mathcal{N}_{0, i}\left(\right.$ or $\mathcal{N}_{0, i}^{+}$) by applying $\mathcal{M}_{i, 0}\left(\right.$ or $\left.\mathcal{M}_{i, 0}^{+}\right)$and 
$\mathcal{M}_{0, i}\left(\operatorname{or} \mathcal{M}_{0, i}^{+}\right)$to the first part,

$$
\begin{aligned}
& P_{n_{1}+n_{2}, n_{2}}^{\left(\alpha, \beta, \gamma+\delta+2 n_{3}+b+1, a\right)}(x, y) \\
& \quad:=P_{n_{1}}^{\left(\beta+\gamma+\delta+a+b+2 n_{2}+2 n_{3}+2, \alpha\right)}(2 x-1)(1-x)^{n_{2}} P_{n_{2}}^{\left(\gamma+\delta+2 n_{3}+b+1, \beta\right)}\left(\frac{2 y}{1-x}-1\right),
\end{aligned}
$$

respectively, and for $\mathcal{O}_{i, 0}\left(\right.$ or $\left.\mathcal{O}_{i, 0}^{+}\right)$by applying $\widetilde{\mathcal{L}}_{i}\left(\right.$ or $\left.\widetilde{\mathcal{L}}_{i}^{+}\right)$to the second part $P_{n_{3}}^{(\delta, \gamma)}\left(\frac{2 z}{1-x-y}-1\right)$ in the polynomial $P_{n_{1}, n_{2}, n_{3}}^{(\alpha, \beta, \gamma, b)}(x, y, z)$ for $1 \leq i \leq 6$.

Theorem 1 The following sparse recurrence relations for $P_{n_{1}, n_{2}, n_{3}}^{(\alpha, \beta, \gamma, a, b)}(x, y, z)$ hold true:

$$
\begin{aligned}
& \mathcal{N}_{0,1} P_{n_{1}, n_{2}, n_{3}}^{(\alpha, \beta, \gamma, a, b)}(x, y, z)=\left(n_{2}+2 n_{3}+\beta+\gamma+\delta+b+2\right) P_{n_{1}, n_{2}-1, n_{3}}^{(\alpha, \beta+1, \gamma, \delta, a, b+1)}(x, y, z), \\
& \mathcal{N}_{0,1}^{+} P_{n_{1}, n_{2}, n_{3}}^{(\alpha, \beta, \gamma, \delta, a, b)}(x, y, z)=\left(n_{2}+1\right) P_{n_{1}, n_{2}+1, n_{3}}^{(\alpha, \beta-1, \gamma, \delta, 1)}(x, y, z), \\
& \mathcal{N}_{0,2} P_{n_{1}, n_{2}, n_{3}}^{(\alpha, \beta, \gamma, b)}(x, y, z)=\left(n_{2}+2 n_{3}+\beta+\gamma+\delta+b+2\right) P_{n_{1}, n_{2}, n_{3}}^{(\alpha, \beta, \gamma, \alpha, 1, b+1)}(x, y, z) \text {, } \\
& \mathcal{N}_{0,2}^{+} P_{n_{1}, n_{2}, n_{3}}^{(\alpha, \beta, \gamma, \delta, a)}(x, y, z)=\left(n_{2}+2 n_{3}+\gamma+\delta+b+1\right) P_{n_{1}, n_{2}, n_{3}}^{(\alpha, \beta, \gamma, a+1, b-1)}(x, y, z), \\
& \mathcal{N}_{0,3} P_{n_{1}, n_{2}, n_{3}}^{(\alpha, \beta, \alpha, b)}(x, y, z)=\left(n_{2}+2 n_{3}+\beta+\gamma+\delta+b+2\right) P_{n_{1}, n_{2}, n_{3}}^{(\alpha, \beta, 1, \gamma, \delta, 1, b)}(x, y, z) \text {, } \\
& \mathcal{N}_{0,3}^{+} P_{n_{1}, n_{2}, n_{3}}^{(\alpha, \beta, \gamma, b)}(x, y, z)=\left(n_{2}+\beta\right) P_{n_{1}, n_{2}, n_{3}}^{(\alpha, \beta-1, \gamma, \delta, a+1, b)}(x, y, z), \\
& \mathcal{N}_{0,4} P_{n_{1}, n_{2}, n_{3}}^{(\alpha, \beta, \gamma, a, b)}(x, y, z)=\left(n_{2}+1\right) P_{n_{1}, n_{2}+1, n_{3}}^{(\alpha, \beta, \gamma, \delta, a-1, b-1)}(x, y, z), \\
& \mathcal{N}_{0,4}^{+} P_{n_{1}, n_{2}, n_{3}}^{(\alpha, \beta, \gamma, \delta, a, b)}(x, y, z)=\left(n_{2}+\beta\right) P_{n_{1}, n_{2}-1, n_{3}}^{(\alpha, \beta, \delta, \delta+1, b+1)}(x, y, z), \\
& \mathcal{N}_{0,5} P_{n_{1}, n_{2}, n_{3}}^{(\alpha, \beta, \gamma, a, b)}(x, y, z)=\left(n_{2}+1\right) P_{n_{1}, n_{2}+1, n_{3}}^{(\alpha, \beta-1, \gamma-1, b)}(x, y, z), \\
& \mathcal{N}_{0,5}^{+} P_{n_{1}, n_{2}, n_{3}}^{(\alpha, \beta, \gamma, \delta, a, b)}(x, y, z)=\left(n_{2}+2 n_{3}+\gamma+\delta+b+1\right) P_{n_{1}, n_{2}-1, n_{3}}^{(\alpha, \beta+1, \gamma, \delta, a+1, b)}(x, y, z), \\
& \mathcal{N}_{0,6} P_{n_{1}, n_{2}, n_{3}}^{(\alpha, \beta, \gamma, b, b)}(x, y, z)=\left(n_{2}+\beta\right) P_{n_{1}, n_{2}, n_{3}}^{(\alpha, \beta-1, \gamma, \delta, a, b+1)}(x, y, z), \\
& \mathcal{N}_{0,6}^{+} P_{n_{1}, n_{2}, n_{3}}^{(\alpha, \beta, \gamma, b)}(x, y, z)=\left(n_{2}+2 n_{3}+\gamma+\delta+b+1\right) P_{n_{1}, n_{2}, n_{3}}^{(\alpha, \beta+1, \gamma, \delta, a, b-1)}(x, y, z) .
\end{aligned}
$$

\section{Moreover,}

$$
\begin{aligned}
& \mathcal{N}_{1,0} P_{n_{1}, n_{2}, n_{3}}^{(\alpha, \beta, \gamma, b)}(x, y, z)=\left(n+n_{2}+n_{3}+e+3\right) P_{n_{1}-1, n_{2}, n_{3}}^{(\alpha+1, \beta+1, b)}(x, y, z), \\
& \mathcal{N}_{1,0}^{+} P_{n_{1}, n_{2}, n_{3}}^{(\alpha, \beta, \gamma, b)}(x, y, z)=\left(n_{1}+1\right) P_{n_{1}+1, n_{2}, n_{3}}^{(\alpha-1, \beta,-1, b)}(x, y, z), \\
& \mathcal{N}_{2,0} P_{n_{1}, n_{2}, n_{3}}^{(\alpha, \beta, \gamma, b)}(x, y, z)=\left(n+n_{2}+n_{3}+e+3\right) P_{n_{1}, n_{2}, n_{3}}^{(\alpha, \beta, a+1, b)}(x, y, z), \\
& \mathcal{N}_{2,0}^{+} P_{n_{1}, n_{2}, n_{3}}^{(\alpha, \beta, \gamma, b)}(x, y, z)=\left(n+n_{2}+n_{3}+e-\alpha+2\right) P_{n_{1}, n_{2}, n_{3}}^{(\alpha, \beta, \gamma, a-1, b)}(x, y, z), \\
& \mathcal{N}_{3,0} P_{n_{1}, n_{2}, n_{3}}^{(\alpha, \beta, \gamma, a, b)}(x, y, z)=\left(n+n_{2}+n_{3}+e+3\right) P_{n_{1}, n_{2}, n_{3}}^{(\alpha+1, \beta, \gamma, \delta, a, b)}(x, y, z), \\
& \mathcal{N}_{3,0}^{+} P_{n_{1}, n_{2}, n_{3}}^{(\alpha, \beta, \gamma, \delta, a, b)}(x, y, z)=\left(n_{1}+\alpha\right) P_{n_{1}, n_{2}, n_{3}}^{(\alpha-1, \beta, \gamma, \delta, a, b)}(x, y, z), \\
& \mathcal{N}_{4,0} P_{n_{1}, n_{2}, n_{3}}^{(\alpha, \beta, \gamma, \delta, a, b)}(x, y, z)=\left(n_{1}+1\right) P_{n_{1}+1, n_{2}, n_{3}}^{(\alpha, \beta, \gamma, \delta, a-1, b)}(x, y, z), \\
& \mathcal{N}_{4,0}^{+} P_{n_{1}, n_{2}, n_{3}}^{(\alpha, \beta, \gamma, \delta, a, b)}(x, y, z)=\left(n_{1}+\alpha\right) P_{n_{1}-1, n_{2}, n_{3}}^{(\alpha, \beta, \gamma, \delta, a+1, b)}(x, y, z), \\
& \mathcal{N}_{5,0} P_{n_{1}, n_{2}, n_{3}}^{(\alpha, \beta, \gamma, \delta, a, b)}(x, y, z)=\left(n_{1}+1\right) P_{n_{1}+1, n_{2}, n_{3}}^{(\alpha-1, \beta, \gamma, a, b)}(x, y, z), \\
& \mathcal{N}_{5,0}^{+} P_{n_{1}, n_{2}, n_{3}}^{(\alpha, \beta, \gamma, \delta, a, b)}(x, y, z)=\left(n+n_{2}+n_{3}+e-\alpha+2\right) P_{n_{1}-1, n_{2}, n_{3}}^{(\alpha+1, \beta, \gamma, a, b)}(x, y, z), \\
& \mathcal{N}_{6,0} P_{n_{1}, n_{2}, n_{3}}^{(\alpha, \beta, \gamma, \delta, a, b)}(x, y, z)=\left(n_{1}+\alpha\right) P_{n_{1}, n_{2}, n_{3}}^{(\alpha-1, \beta, \gamma, \delta, a+1, b)}(x, y, z),
\end{aligned}
$$




$$
\mathcal{N}_{6,0}^{+} P_{n_{1}, n_{2}, n_{3}}^{(\alpha, \beta, \gamma, a, b)}(x, y, z)=\left(n+n_{2}+n_{3}+e-\alpha+2\right) P_{n_{1}, n_{2}, n_{3}}^{(\alpha+1, \gamma, \alpha-1, b)}(x, y, z)
$$

Also,

$$
\begin{aligned}
& \mathcal{O}_{1,0} P_{n_{1}, n_{2}, n_{3}}^{(\alpha, \beta, \delta, b)}(x, y, z)=\left(n_{3}+\delta+\gamma+1\right) P_{n_{1}, n_{2}, n_{3}-1}^{(\alpha, \beta, \gamma+1, \delta+1, a, b)}(x, y, z), \\
& \mathcal{O}_{1,0}^{+} P_{n_{1}, n_{2}, n_{3}}^{(\alpha, \beta, \delta, b)}(x, y, z)=\left(n_{3}+1\right) P_{n_{1}, n_{2}, n_{3}+1}^{(\alpha, \beta, \gamma-1, \delta-1, a, b)}(x, y, z), \\
& \mathcal{O}_{2,0} P_{n_{1}, n_{2}, n_{3}}^{(\alpha, \beta, \gamma, a, b)}(x, y, z)=\left(n_{3}+\delta+\gamma+1\right) P_{n_{1}, n_{2}, n_{3}}^{(\alpha, \beta, \gamma, \delta+a, b-1)}(x, y, z) \text {, } \\
& \mathcal{O}_{2,0}^{+} P_{n_{1}, n_{2}, n_{3}}^{(\alpha, \beta, \gamma, \delta, a, b)}(x, y, z)=\left(n_{3}+\delta\right) P_{n_{1}, n_{2}, n_{3}}^{(\alpha, \beta,-1, a, b+1)}(x, y, z), \\
& \mathcal{O}_{3,0} P_{n_{1}, n_{2}, n_{3}}^{(\alpha, \beta, \gamma, \delta, a, b)}(x, y, z)=\left(n_{3}+\delta+\gamma+1\right) P_{n_{1}, n_{2}, n_{3}}^{(\alpha, \beta, \gamma+1, \delta, a, b-1)}(x, y, z) \text {, } \\
& \mathcal{O}_{3,0}^{+} P_{n_{1}, n_{2}, n_{3}}^{(\alpha, \beta, \gamma, \delta, b)}(x, y, z)=\left(n_{3}+\gamma\right) P_{n_{1}, n_{2}, n_{3}}^{(\alpha, \beta,-1, \delta, a, b+1)}(x, y, z), \\
& \mathcal{O}_{4,0} P_{n_{1}, n_{2}, n_{3}}^{(\alpha, \beta, \gamma, \delta, a, b)}(x, y, z)=\left(n_{3}+1\right) P_{n_{1}, n_{2}, n_{3}+1}^{(\alpha, \beta, \gamma, \delta-1, a, b-1)}(x, y, z), \\
& \mathcal{O}_{4,0}^{+} P_{n_{1}, n_{2}, n_{3}}^{(\alpha, \beta, \gamma, \delta, a, b)}(x, y, z)=\left(n_{3}+\gamma\right) P_{n_{1}, n_{2}, n_{3}-1}^{(\alpha, \beta, \gamma, \delta+a, b+1)}(x, y, z), \\
& \mathcal{O}_{5,0} P_{n_{1}, n_{2}, n_{3}}^{(\alpha, \beta, \gamma, \delta, a, b)}(x, y, z)=\left(n_{3}+1\right) P_{n_{1}, n_{2}, n_{3}+1}^{(\alpha, \beta, \gamma-1, \delta, a, b-1)}(x, y, z), \\
& \mathcal{O}_{5,0}^{+} P_{n_{1}, n_{2}, n_{3}}^{(\alpha, \beta, \gamma, \delta, a, b)}(x, y, z)=\left(n_{3}+\delta\right) P_{n_{1}, n_{2}, n_{3}-1}^{(\alpha, \beta,+1, \delta, a, b+1)}(x, y, z) \text {, } \\
& \mathcal{O}_{6,0} P_{n_{1}, n_{2}, n_{3}}^{(\alpha, \beta, \delta, a, b)}(x, y, z)=\left(n_{3}+\gamma\right) P_{n_{1}, n_{2}, n_{3}}^{(\alpha, \beta, \gamma+1, a, b)}(x, y, z), \\
& \mathcal{O}_{6,0}^{+} P_{n_{1}, n_{2}, n_{3}}^{(\alpha, \beta, \gamma, \delta, a, b)}(x, y, z)=\left(n_{3}+\delta\right) P_{n_{1}, n_{2}, n_{3}}^{(\alpha, \beta, \gamma+1, \delta-1, a, b)}(x, y, z) \text {, }
\end{aligned}
$$

where $n_{1}+n_{2}+n_{3}=n$ and $\alpha+\beta+\gamma+\delta+a+b=e$.

Proof Let us denote

$$
P_{n_{1}, n_{2}, n_{3}}^{(\alpha, \beta, \gamma, a, b)}(x, y, z):=P_{n_{1}+n_{2}, n_{2}}^{\left(\alpha, \beta, \gamma+\delta n_{3}+b+1, a\right)}(x, y)(1-x-y)^{n_{3}} P_{n_{3}}^{(\delta, \gamma)}\left(\frac{2 z}{1-x-y}-1\right)
$$

If we apply $\mathcal{M}_{0,1}$ to both sides of $P_{n_{1}, n_{2}, n_{3}}^{(\alpha, \beta, \gamma, a, b)}(x, y, z)$, we have

$$
\mathcal{N}_{0,1} P_{n_{1}, n_{2}, n_{3}}^{(\alpha, \beta, \gamma, a, b)}(x, y, z)=(1-x-y)^{n_{3}} P_{n_{3}}^{(\delta, \gamma)}\left(\frac{2 z}{1-x-y}-1\right) \mathcal{M}_{0,1} P_{n_{1}+n_{2}, n_{2}}^{\left(\alpha, \beta, \gamma+\delta+2 n_{3}+b+1, a\right)}(x, y)
$$

From the relation

$$
\mathcal{M}_{0,1} P_{n, k}^{(a, b, c, d)}(x, y)=(k+b+c+1) P_{n-1, k-1}^{(a, b+1, c+1, d)}(x, y)
$$

it follows that

$$
\mathcal{N}_{0,1} P_{n_{1}, n_{2}, n_{3}}^{(\alpha, \beta, \gamma, \delta, a, b}(x, y, z)=\left(n_{2}+2 n_{3}+\beta+\gamma+\delta+b+2\right) P_{n_{1}, n_{2}-1, n_{3}}^{(\alpha, \beta+1, \gamma, b+1)}(x, y, z) .
$$

Similarly, in view of $\mathcal{M}_{i, 0}, \mathcal{M}_{i, 0}^{+}, \mathcal{M}_{0, i}, \mathcal{M}_{0, i}^{+}, \widetilde{\mathcal{L}}_{i}$ and $\widetilde{\mathcal{L}}_{i}^{+}, 1 \leq i \leq 6$, the other recurrence relations are obtained.

From these relations we have

$$
\mathcal{N}_{0,1}^{+} \mathcal{N}_{0,1} P_{n_{1}, n_{2}, n_{3}}^{(\alpha, \beta-1, \gamma, \delta, a, b-1)}(x, y, z)=n_{2}\left(n_{2}+2 n_{3}+\beta+\gamma+\delta+b\right) P_{n_{1}, n_{2}, n_{3}}^{(\alpha, \beta-1, \gamma, \delta, a, b-1)}(x, y, z)
$$




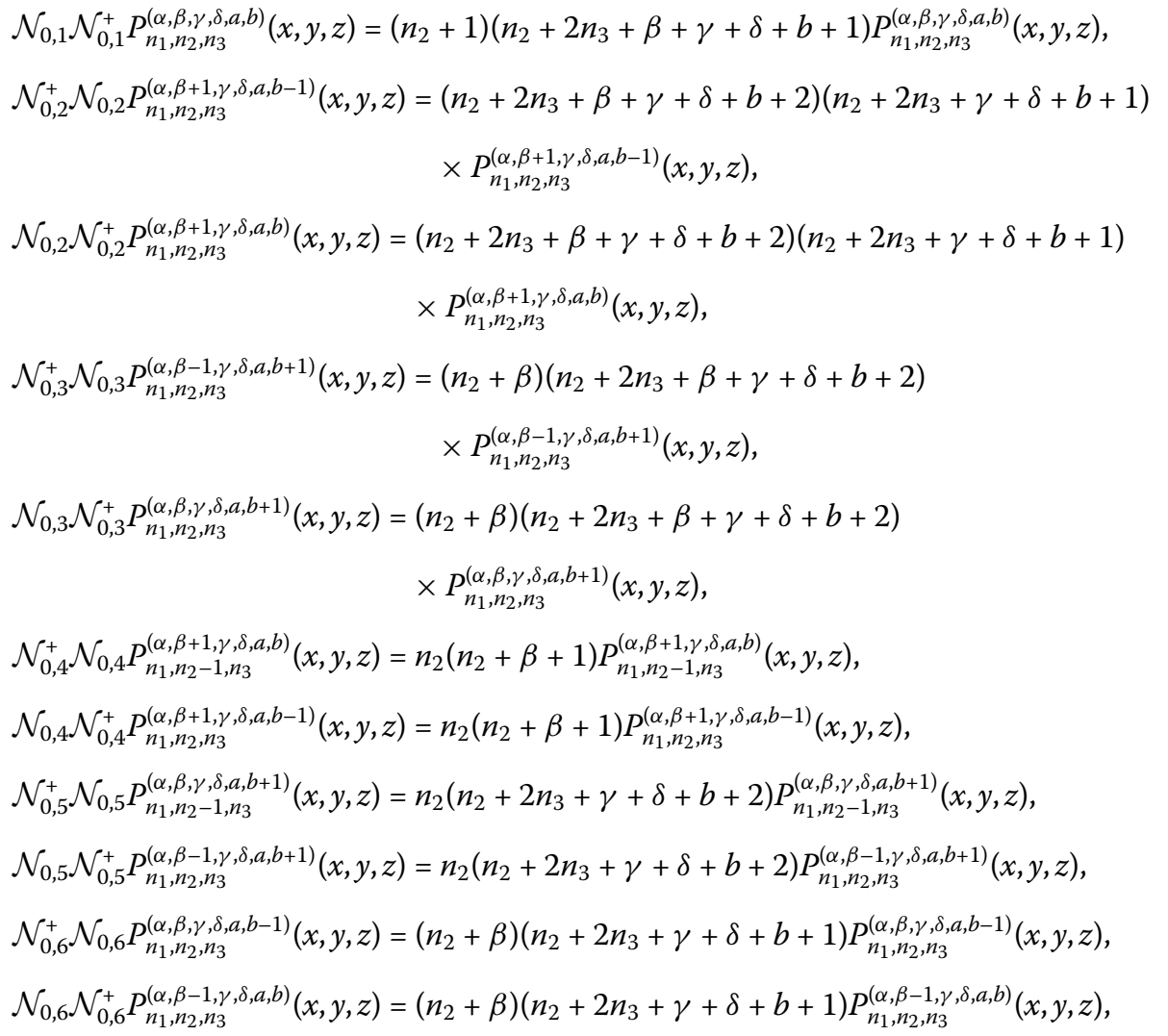

and

$$
\begin{aligned}
& \mathcal{N}_{1,0}^{+} \mathcal{N}_{1,0} P_{n_{1}, n_{2}, n_{3}}^{(\alpha-1, \beta, \delta, a, b-1)}(x, y, z)=n_{1}\left(n+n_{2}+n_{3}+e+1\right) P_{n_{1}, n_{2}, n_{3}}^{(\alpha-1, \beta, \gamma, \delta, a, b-1)}(x, y, z), \\
& \mathcal{N}_{1,0} \mathcal{N}_{1,0}^{+} P_{n_{1}, n_{2}, n_{3}}^{(\alpha, \beta, \gamma, \delta, a, b)}(x, y, z)=\left(n_{1}+1\right)\left(n+n_{2}+n_{3}+e+2\right) P_{n_{1}, n_{2}, n_{3}}^{(\alpha, \beta, \gamma, \delta, a, b)}(x, y, z), \\
& \mathcal{N}_{2,0}^{+} \mathcal{N}_{2,0} P_{n_{1}, n_{2}, n_{3}}^{(\alpha+1, \beta, \gamma, a, b-1)}(x, y, z)=\left(n+n_{2}+n_{3}+e+3\right)\left(n+n_{2}+n_{3}+e-\alpha+2\right) \\
& \times P_{n_{1}, n_{2}, n_{3}}^{(\alpha+1, \beta, \gamma, b-1)}(x, y, z), \\
& \mathcal{N}_{2,0} \mathcal{N}_{2,0}^{+} P_{n_{1}, n_{2}, n_{3}}^{(\alpha+1, \beta, \gamma, a, b)}(x, y, z)=\left(n+n_{2}+n_{3}+e+3\right)\left(n+n_{2}+n_{3}+e-\alpha+2\right) \\
& \times P_{n_{1}, n_{2}, n_{3}}^{(\alpha+1, \beta, \delta, a, b)}(x, y, z), \\
& \mathcal{N}_{3,0}^{+} \mathcal{N}_{3,0} P_{n_{1}, n_{2}, n_{3}}^{(\alpha-1, \beta, \gamma, a+1, b)}(x, y, z)=\left(n_{1}+\alpha\right)\left(n+n_{2}+n_{3}+e+3\right) P_{n_{1}, n_{2}, n_{3}}^{(\alpha, \beta, \gamma, a+1, b)}(x, y, z), \\
& \mathcal{N}_{3,0} \mathcal{N}_{3,0}^{+} P_{n_{1}, n_{2}, n_{3}}^{(\alpha, \beta, \gamma, a+1, b)}(x, y, z)=\left(n_{1}+\alpha\right)\left(n+n_{2}+n_{3}+e+3\right) P_{n_{1}, n_{2}, n_{3}}^{(\alpha, \beta, \alpha+1, b)}(x, y, z), \\
& \mathcal{N}_{4,0}^{+} \mathcal{N}_{4,0} P_{n_{1}-1, n_{2}, n_{3}}^{(\alpha+1, \beta, \gamma, \delta, b)}(x, y, z)=n_{1}\left(n_{1}+\alpha+1\right) P_{n_{1}-1, n_{2}, n_{3}}^{(\alpha+1, \beta, \gamma, \delta, a, b)}(x, y, z), \\
& \mathcal{N}_{4,0} \mathcal{N}_{4,0}^{+} P_{n_{1}, n_{2}, n_{3}}^{(\alpha+1, \beta, \delta, a, b-1)}(x, y, z)=n_{1}\left(n_{1}+\alpha+1\right) P_{n_{1}, n_{2}, n_{3}}^{(\alpha+1, \beta, \gamma, \delta, a, b-1)}(x, y, z), \\
& \mathcal{N}_{5,0}^{+} \mathcal{N}_{5,0} P_{n_{1}-1, n_{2}, n_{3}}^{(\alpha, \beta, \gamma, \delta, a+1, b)}(x, y, z)=n_{1}\left(n+n_{2}+n_{3}+e-\alpha+3\right) P_{n_{1}-1, n_{2}, n_{3}}^{(\alpha, \beta, \gamma, \delta, a+1, b)}(x, y, z), \\
& \mathcal{N}_{5,0} \mathcal{N}_{5,0}^{+} P_{n_{1}, n_{2}, n_{3}}^{(\alpha, \beta, a+1, b)}(x, y, z)=n_{1}\left(n+n_{2}+n_{3}+e-\alpha+3\right) P_{n_{1}, n_{2}, n_{3}}^{(\alpha-1, \beta, a+1, b)}(x, y, z), \\
& \mathcal{N}_{6,0}^{+} \mathcal{N}_{6,0} P_{n_{1}, n_{2}, n_{3}}^{(\alpha, \beta, \gamma, a, b-1)}(x, y, z)=\left(n_{1}+\alpha\right)\left(n+n_{2}+n_{3}+e-\alpha+2\right) P_{n_{1}, n_{2}, n_{3}}^{(\alpha, \beta, \gamma, \delta, a-1)}(x, y, z), \\
& \mathcal{N}_{6,0} \mathcal{N}_{6,0}^{+} P_{n_{1}, n_{2}, n_{3}}^{(\alpha-1, \beta, \alpha, b)}(x, y, z)=\left(n_{1}+\alpha\right)\left(n+n_{2}+n_{3}+e-\alpha+2\right) P_{n_{1}, n_{2}, n_{3}}^{(\alpha-\beta, \delta, b)}(x, y, z),
\end{aligned}
$$


and

$$
\begin{aligned}
& \mathcal{O}_{1,0}^{+} \mathcal{O}_{1,0} P_{n_{1}, n_{2}, n_{3}}^{(\alpha, \beta-1, a, b)}(x, y, z)=n_{3}\left(n_{3}+\gamma+\delta-1\right) P_{n_{1}, n_{2}, n_{3}}^{(\alpha, \beta, \gamma-1, \delta-1, a, b)}(x, y, z), \\
& \mathcal{O}_{1,0} \mathcal{O}_{1,0}^{+} P_{n_{1}, n_{2}, n_{3}}^{(\alpha, \beta, \gamma, a, b)}(x, y, z)=\left(n_{3}+1\right)\left(n_{3}+\gamma+\delta\right) P_{n_{1}, n_{2}, n_{3}}^{(\alpha, \beta, \gamma, a, b)}(x, y, z), \\
& \mathcal{O}_{2,0}^{+} \mathcal{O}_{2,0} P_{n_{1}, n_{2}, n_{3}}^{(\alpha, \beta, \gamma+1, a, b)}(x, y, z)=\left(n_{3}+\delta\right)\left(n_{3}+\gamma+\delta+1\right) P_{n_{1}, n_{2}, n_{3}}^{(\alpha, \beta, \gamma+1, a, b)}(x, y, z) \text {, } \\
& \mathcal{O}_{2,0} \mathcal{O}_{2,0}^{+} P_{n_{1}, n_{2}, n_{3}}^{(\alpha, \beta, \gamma+1, \delta, a, b)}(x, y, z)=\left(n_{3}+\delta\right)\left(n_{3}+\gamma+\delta+1\right) P_{n_{1}, n_{2}, n_{3}}^{(\alpha, \beta, \gamma+1, \delta, a, b)}(x, y, z), \\
& \mathcal{O}_{3,0}^{+} \mathcal{O}_{3,0} P_{n_{1}, n_{2}, n_{3}}^{(\alpha, \beta, \gamma-1, \delta+1, a, b)}(x, y, z)=\left(n_{3}+\gamma\right)\left(n_{3}+\gamma+\delta+1\right) P_{n_{1}, n_{2}, n_{3}}^{(\alpha, \beta, \gamma-1, a, b)}(x, y, z) \text {, } \\
& \mathcal{O}_{3,0} \mathcal{O}_{3,0}^{+} P_{n_{1}, n_{2}, n_{3}}^{(\alpha, \beta, \gamma, 1, a, b)}(x, y, z)=\left(n_{3}+\gamma\right)\left(n_{3}+\gamma+\delta+1\right) P_{n_{1}, n_{2}, n_{3}}^{(\alpha, \beta, \gamma, \delta, a, b)}(x, y, z) \text {, } \\
& \mathcal{O}_{4,0}^{+} \mathcal{O}_{4,0} P_{n_{1}, n_{2}, n_{3}-1}^{(\alpha, \beta, \gamma+1, \delta, a, b)}(x, y, z)=n_{3}\left(n_{3}+\gamma+1\right) P_{n_{1}, n_{2}, n_{3}-1}^{(\alpha, \beta, \gamma+1, a, b)}(x, y, z), \\
& \mathcal{O}_{4,0} \mathcal{O}_{4,0}^{+} P_{n_{1}, n_{2}, n_{3}}^{(\alpha, \beta, \gamma+1, \delta, a)}(x, y, z)=n_{3}\left(n_{3}+\gamma+1\right) P_{n_{1}, n_{2}, n_{3}}^{(\alpha, \beta, \gamma+1, \delta-1, a, b)}(x, y, z) \text {, } \\
& \mathcal{O}_{5,0}^{+} \mathcal{O}_{5,0} P_{n_{1}, n_{2}, n_{3}-1}^{(\alpha, \beta, \gamma, \delta+a, b)}(x, y, z)=n_{3}\left(n_{3}+\delta+1\right) P_{n_{1}, n_{2}, n_{3}-1}^{(\alpha, \beta, \gamma, \alpha+1, a, b)}(x, y, z), \\
& \mathcal{O}_{5,0} \mathcal{O}_{5,0}^{+} P_{n_{1}, n_{2}, n_{3}}^{(\alpha, \beta, \gamma-1, \delta+1, a, b)}(x, y, z)=n_{3}\left(n_{3}+\delta+1\right) P_{n_{1}, n_{2}, n_{3}}^{(\alpha, \beta, \gamma-1, \delta+1, a, b)}(x, y, z) \text {, } \\
& \mathcal{O}_{6,0}^{+} \mathcal{O}_{6,0} P_{n_{1}, n_{2}, n_{3}}^{(\alpha, \beta, \gamma, \delta-1, a, b)}(x, y, z)=\left(n_{3}+\gamma\right)\left(n_{3}+\delta\right) P_{n_{1}, n_{2}, n_{3}}^{(\alpha, \beta, \gamma, \delta-1, a, b)}(x, y, z), \\
& \mathcal{O}_{6,0} \mathcal{O}_{6,0}^{+} P_{n_{1}, n_{2}, n_{3}}^{(\alpha, \beta, \gamma-1, \delta, a, b)}(x, y, z)=\left(n_{3}+\gamma\right)\left(n_{3}+\delta\right) P_{n_{1}, n_{2}, n_{3}}^{(\alpha, \beta, \gamma-1, \delta, a, b)}(x, y, z) .
\end{aligned}
$$

Theorem 2 The polynomials $P_{n_{1}, n_{2}, n_{3}}^{(\alpha, \beta, b, b)}(x, y, z)$ defined in (11) satisfy the following second order partial differential equations:

$$
\begin{aligned}
T_{1} u:= & x(1-x) u_{x x}+y(1-y) u_{y y}+z(1-z) u_{z z}-2 x z u_{x z}-2 y z u_{y z}-2 x y u_{x y} \\
& +(\alpha+1-(\alpha+\beta+\gamma+\delta+a+b+4) x) u_{x} \\
& +\left(\beta+1-(\alpha+\beta+\gamma+\delta+4) y+\frac{(a+b) x y-b y}{1-x}\right) u_{y} \\
& +\left(\gamma+1-(\alpha+\beta+\gamma+\delta+4) z+\frac{(a+b) x z}{1-x}+\frac{b y z}{(1-x)(1-x-y)}\right) u_{z} \\
& +\left(n(n+\alpha+\beta+\gamma+\delta+a+b+3)-\frac{a\left(n_{2}+n_{3}\right)}{1-x}-\frac{n_{3} b}{1-x-y}\right) u
\end{aligned}
$$$$
=0 \text {, }
$$

$$
\begin{aligned}
T_{2} u:= & y(1-x-y) u_{y y}-2 y z u_{y z}+\frac{y z^{2}}{1-x-y} u_{z z} \\
& +((\beta+1)(1-x-y)-(\gamma+\delta+b+2) y) u_{y} \\
& +\frac{1}{1-x-y}((\gamma+\delta+b+2) y z-(\beta+1) z(1-x-y)) u_{z} \\
& +\left((\beta+1) n_{3}+n_{2}\left(n_{2}+2 n_{3}+\beta+\gamma+\delta+b+2\right)-\frac{n_{3}\left(\gamma+\delta+b+n_{3}+1\right) y}{1-x-y}\right) u
\end{aligned}
$$$$
=0 \text {, }
$$

$T_{3} u:=z(1-x-y-z) u_{z z}+[(\gamma+1)(1-x-y-z)-(\delta+1) z] u_{z}+n_{3}\left(n_{3}+\gamma+\delta+1\right) u$

$$
=0 \text {, }
$$


and

$$
\begin{aligned}
T_{4} u:= & x(1-x) u_{x x}+\frac{x y^{2}}{1-x} u_{y y}+\frac{x z^{2}}{1-x} u_{z z}-2 x y u_{x y}-2 x z u_{x z}+\frac{2 x y z}{1-x} u_{y z} \\
& +(\alpha+1-(\alpha+\beta+\gamma+\delta+a+b+4) x) u_{x} \\
& -\frac{y}{1-x}(\alpha+1-(\alpha+\beta+\gamma+\delta+a+b+4) x) u_{y} \\
& -\frac{z}{1-x}(\alpha+1-(\alpha+\beta+\gamma+\delta+a+b+4) x) u_{z} \\
& +(n(n+\alpha+\beta+\gamma+\delta+a+b+3) \\
& \left.-\frac{\left(n_{2}+n_{3}\right)\left(n_{2}+n_{3}+\beta+\gamma+\delta+a+b+2\right)}{1-x}\right) u \\
= & 0 .
\end{aligned}
$$

Proof The first equation is obtained from

$$
-\mathcal{N}_{10} \mathcal{N}_{10}^{+}-\frac{1}{1-x} \mathcal{N}_{01} \mathcal{N}_{01}^{+}-\frac{1}{1-x-y} \mathcal{O}_{10} \mathcal{O}_{10}^{+}
$$

and the second and third equations follow from $\mathcal{N}_{01} \mathcal{N}_{01}^{+}$and $\mathcal{O}_{10} \mathcal{O}_{10}^{+}$, respectively. From

$$
T_{1} u-\frac{1}{1-x} T_{2} u-\frac{1}{1-x-y} T_{3} u=0,
$$

we obtain the last equation.

Remark 3 For $a=b=0$, the first equation reduces to the partial differential equation [3] for the polynomials $P_{n_{1}, n_{2}, n_{3}}^{(\alpha, \beta)}(x, y, z)$ defined in (10)

$$
\begin{aligned}
& x(1-x) u_{x x}+y(1-y) u_{y y}+z(1-z) u_{z z}-2 x z u_{x z}-2 y z u_{y z}-2 x y u_{x y} \\
& \quad+(\alpha+1-(\alpha+\beta+\gamma+\delta+4) x) u_{x}+(\beta+1-(\alpha+\beta+\gamma+\delta+4) y) u_{y} \\
& \quad+(\gamma+1-(\alpha+\beta+\gamma+\delta+4) z) u_{z}+n(n+\alpha+\beta+\gamma+\delta+3) u \\
& =0 .
\end{aligned}
$$

Remark 4 For the second order differential equations in the first set given above, the first, second and the last two equations give the second order equation in the form of (21). The third, fourth, seventh and eighth equations give Eq. (21) multiplied by $y$. The fifth, sixth, ninth and tenth equations give Eq. (21) multiplied by $1-x-y$. For the second order differential equations in the second set, the first, second and the last two equations give the second order equation in the form of (23). The third, fourth, seventh and eighth equations give Eq. (23) multiplied by $x$. The fifth, sixth, ninth and tenth equations give Eq. (23) multiplied by $1-x$. Moreover, for the second order differential equations given in the third set, the first, second and the last two equations give the second order equation in the form of (22). The third, fourth, seventh and eighth equations give Eq. (22) multiplied by $z$. The fifth, sixth, ninth and tenth equations give Eq. (22) multiplied by $1-x-y-z$. 
Remark 5 The partial differential equation (23) has also a monic solution, given by

$$
\begin{aligned}
\tilde{P}_{n_{1}, n_{2}, n_{3}}^{(\alpha, \beta, \gamma, \delta, a b}(x, y, z)= & \frac{(-1)^{n_{1}} \Gamma\left(\alpha+n_{1}+1\right) \Gamma\left(\alpha+\beta+\gamma+\delta+a+b+n+n_{2}+n_{3}+3\right)}{\Gamma(\alpha+1) \Gamma(\alpha+\beta+\gamma+\delta+a+b+2 n+3)} \\
& \times y^{n_{2}} z^{n_{3}}{ }_{2} F_{1}\left(\begin{array}{c}
-n_{1}, \alpha+\beta+\gamma+\delta+a+b+n+n_{2}+n_{3}+3 \\
\alpha+1
\end{array} \mid x\right),
\end{aligned}
$$

which can be written as

$$
\begin{aligned}
\tilde{P}_{n_{1}, n_{2}, n_{3}}^{(\alpha, \gamma, \gamma)}(x, y, z)= & \frac{n_{1} ! \Gamma\left(\alpha+\beta+\gamma+\delta+a+b+n+n_{2}+n_{3}+3\right)}{\Gamma(\alpha+\beta+\gamma+\delta+a+b+2 n+3)} \\
& \times y^{n_{2}} z^{n_{3}} \widetilde{P}_{n_{1}}^{\left(\beta+\gamma+\delta+a+b+2 n_{2}+2 n_{3}+2, \alpha\right)}(x)
\end{aligned}
$$

in terms of shifted Jacobi polynomials.

Theorem 3 The following connection relation holds true:

$$
\begin{aligned}
& P_{\substack{(\alpha, \beta, \gamma, \delta, a, b) \\
n_{1}, n_{3}}}^{(\alpha, y, z)} \\
& =\sum_{m=0}^{n_{1}} \frac{(-1)^{m}(2 n-2 m+e-\alpha+\xi+3) \Gamma\left(n+n_{2}+n_{3}+e-\alpha+3\right)(\alpha-\xi)_{m}}{\Gamma\left(n+n_{2}+n_{3}+e+3\right) \Gamma(2 n-m+e-\alpha+\xi+4)} \\
& \quad \times \frac{\Gamma(2 n-m+e+3) \Gamma\left(n+n_{2}+n_{3}-m+e-\alpha+\xi+3\right)}{m ! \Gamma\left(n+n_{2}+n_{3}-m+e-\alpha+3\right)} P_{n_{1}-m, n_{2}, n_{3}}^{(\xi, \beta, \gamma, \delta, a, b)}(x, y, z),
\end{aligned}
$$

where $P_{n_{1}, n_{2}, n_{3}}^{(\alpha, \beta, a)}(x, y, z)$ are defined in $(11), e=\alpha+\beta+\gamma+\delta+a+b, n=n_{1}+n_{2}+n_{3}$, and $(\lambda)_{n}$ denotes the Pochhammer symbol defined by $(\lambda)_{n}=\lambda(\lambda+1) \cdots(\lambda+n-1) ; n \in \mathbb{N}_{0},(\lambda)_{0}=1$.

Proof It follows from the connection relation between the univariate Jacobi polynomials that $[2,5]$

$$
\begin{aligned}
P_{n}^{(\alpha, \beta)}(x)= & \sum_{m=0}^{n} \frac{(-1)^{n-m}(2 m+\alpha+\delta+1) \Gamma(n+\alpha+1) \Gamma(n+m+\alpha+\beta+1)}{\Gamma(m+\alpha+1) \Gamma(n+\alpha+\beta+1)} \\
& \times \frac{\Gamma(m+\alpha+\delta+1)(\beta-\delta)_{n-m}}{\Gamma(n+m+\alpha+\delta+2)(n-m) !} P_{m}^{(\alpha, \delta)}(x) .
\end{aligned}
$$

Theorem 4 The following connection relation holds true:

$$
\begin{aligned}
& P_{n_{1}, n_{2}, n_{3}}^{(\alpha, \beta, \gamma, a, b)}(x, y, z) \\
& =\sum_{k_{1}=0 k_{2}=0 k_{3}=0}^{n_{1}} \sum_{2}^{n_{3}} \frac{\left(k_{1}+2 n_{2}+2 n_{3}+\beta+\gamma+\delta+a+b+3\right)_{n_{1}-k_{1}}\left(n_{1}+2 n_{2}+2 n_{3}+e+3\right)_{k_{1}}}{\left(n_{1}-k_{1}\right) !\left(n_{2}-k_{2}\right) !\left(n_{3}-k_{3}\right) !\left(k_{1}+2 k_{2}+2 k_{3}+\phi+\theta+\xi+\eta+a+b+3\right)_{k_{1}}} \\
& \quad \times \frac{\left(k_{2}+2 n_{3}+\gamma+\delta+b+2\right)_{n_{2}-k_{2}}\left(n_{2}+2 n_{3}+\beta+\gamma+\delta+b+2\right)_{k_{2}}\left(k_{3}+\delta+1\right)_{n_{3}-k_{3}}\left(n_{3}+\gamma+\delta+1\right)_{k_{3}}}{\left(k_{2}+2 k_{3}+\theta+\xi+\eta+b+2\right)_{k_{2}}\left(k_{3}+\xi+\eta+1\right)_{k_{3}}} \\
& \quad \times{ }_{3} F_{2}\left(\begin{array}{c}
k_{1}-n_{1}, n_{1}+2 n_{2}+2 n_{3}+k_{1}+e+3, k_{1}+2 k_{2}+2 k_{3}+\theta+\xi+\eta+a+b+3 \\
2 k_{1}+2 k_{2}+2 k_{3}+\phi+\theta+\xi+\eta+a+b+4, k_{1}+2 n_{2}+2 n_{3}+\beta+\gamma+\delta+a+b+3
\end{array} \mid 1\right) \\
& \quad \times{ }_{3} F_{2}\left(\begin{array}{c}
k_{2}-n_{2}, n_{2}+2 n_{3}+k_{2}+\beta+\gamma+\delta+b+2, k_{2}+2 k_{3}+\xi+\eta+b+2 \\
2 k_{2}+2 k_{3}+\theta+\xi+\eta+b+3, k_{2}+2 n_{3}+\gamma+\delta+b+2
\end{array}\right)
\end{aligned}
$$




$$
\begin{aligned}
& \times{ }_{3} F_{2}\left(\begin{array}{c}
k_{3}-n_{3}, n_{3}+k_{3}+\gamma+\delta+1, k_{3}+\xi+1 \\
2 k_{3}+\xi+\eta+2, k_{3}+\delta+1
\end{array} \mid 1\right) \\
& \times(1-x)^{n_{2}-k_{2}}(1-x-y)^{n_{3}-k_{3}} P_{k_{1}, k_{2}, k_{3}}^{(\phi, \theta, \eta, \xi, a, b)}(x, y, z),
\end{aligned}
$$

where $P_{n_{1}, n_{2}, n_{3}}^{(\alpha, \beta, \gamma, \delta)}(x, y, z)$ are defined in $(11)$.

Proof The result is a consequence of the connection relation between univariate Jacobi polynomials (see [7] and [4])

$$
\begin{aligned}
& P_{n}^{(\alpha, \beta)}(x)=\sum_{k=0}^{n} \frac{(k+\alpha+1)_{n-k}(n+\alpha+\beta+1)_{k}}{(n-k) !(k+\gamma+\delta+1)_{k}} \\
& \times{ }_{3} F_{2}\left(\begin{array}{c|c}
k-n, n+k+\alpha+\beta+1, k+\gamma+1 \\
2 k+\gamma+\delta+2, k+\alpha+1 & 1
\end{array}\right) P_{k}^{(\gamma, \delta)}(x) .
\end{aligned}
$$

Theorem 5 For $n \geq 0$, the three term recurrence relation holds:

$$
\begin{aligned}
x P_{n_{1}, n_{2}, n_{3}}^{(\alpha, \beta, \gamma, \delta, a)}(x, y, z)= & \frac{\left(n_{1}+1\right)\left(e+n+n_{2}+n_{3}+3\right)}{(e+2 n+3)(e+2 n+4)} P_{n_{1}+1, n_{2}, n_{3}}^{(\alpha, \beta, \gamma, \delta, a, b)}(x, y, z) \\
& +\frac{\left(\alpha+2 n_{1}+1\right)(e+2 n+2)-2 n_{1}\left(\alpha+n_{1}\right)}{(e+2 n+2)(e+2 n+4)} P_{n_{1}, n_{2}, n_{3}}^{(\alpha, \beta, a, b)}(x, y, z) \\
& +\frac{\left(n+n_{2}+n_{3}+e-\alpha+2\right)\left(\alpha+n_{1}\right)}{(e+2 n+2)(e+2 n+3)} P_{n_{1}-1, n_{2}, n_{3}}^{(\alpha, \beta, \gamma, \delta, a, b)}(x, y, z),
\end{aligned}
$$

where $n=n_{1}+n_{2}+n_{3}$ and $e=\alpha+\beta+\gamma+\delta+a+b$.

Proof It is enough to use the three term recurrence relation [10, p. 263, Eq. (1)] for the univariate Jacobi polynomials.

\section{Sparse recurrence relations for $P_{n_{1}, n_{2}, n_{3}}^{(\alpha, \beta)}(x, y, z)$}

By combining the results given in the previous section, it is possible to derive sparse recurrence relations between Koornwinder polynomials in three variables with different parameters and their partial derivatives. We denote $w=1-x-y-z$ in the following results.

Corollary 6 The partial derivatives of $P_{n_{1}, n_{2}, n_{3}}^{(\alpha, \beta, \delta)}(x, y, z)$ can be expressed in terms of Koornwinder polynomials in three variables with incremented parameters as follows:

$$
\begin{aligned}
&\left(2 n_{2}+\right.\left.2 n_{3}+\beta+\gamma+\delta+2\right)\left(\frac{\partial}{\partial x}-\frac{\partial}{\partial y}\right) P_{n_{1}, n_{2}, n_{3}}^{(\alpha, \beta, \gamma, \delta)}(x, y, z) \\
&=\left(n_{2}+2 n_{3}+\beta+\gamma+\delta+2\right)\left(n+n_{2}+n_{3}+\alpha+\beta+\gamma+\delta+3\right) P_{n_{1}-1, n_{2}, n_{3}}^{(\alpha+1, \beta+1, \gamma)}(x, y, z) \\
&-\left(n_{1}+2 n_{2}+2 n_{3}+\beta+\gamma+\delta+2\right)\left(n_{2}+2 n_{3}+\gamma+\delta+1\right) P_{n_{1}, n_{2}-1, n_{3}}^{(\alpha+1, \beta+1, \gamma)}(x, y, z), \\
&\left(2 n_{3}+\gamma+\delta+1\right)\left(\frac{\partial}{\partial z}-\frac{\partial}{\partial y}\right) P_{n_{1}, n_{2}, n_{3}}^{(\alpha, \beta, \gamma, \delta)}(x, y, z) \\
&=\left(n_{3}+\delta\right)\left(n_{2}+2 n_{3}+\gamma+\delta+1\right) P_{n_{1}, n_{2}, n_{3}-1}^{(\alpha, \beta+1, \gamma+1, \delta)}(x, y, z) \\
& \quad-\left(n_{2}+2 n_{3}+\beta+\gamma+\delta+2\right)\left(n_{3}+\gamma+\delta+1\right) P_{n_{1}, n_{2}-1, n_{3}}^{(\alpha, \beta+1, \gamma+1, \delta)}(x, y, z), \\
& \frac{\partial}{\partial z} P_{n_{1}, n_{2}, n_{3}}^{(\alpha, \beta, \gamma, \delta)}(x, y, z)=\left(n_{3}+\gamma+\delta+1\right) P_{n_{1}, n_{2}, n_{3}-1}^{(\alpha, \beta, \gamma+1, \delta}(x, y, z),
\end{aligned}
$$


and

$$
\begin{aligned}
\left(2 n_{2}+\right. & \left.2 n_{3}+\beta+\gamma+\delta+2\right) \frac{\partial}{\partial z}\left(\frac{\partial}{\partial x}-\frac{\partial}{\partial y}\right) P_{n_{1}, n_{2}, n_{3}}^{(\alpha, \beta, \gamma}(x, y, z) \\
= & \left(n_{2}+2 n_{3}+\beta+\gamma+\delta+2\right)\left(n+n_{2}+n_{3}+\alpha+\beta+\gamma+\delta+3\right) \\
& \times\left(n_{3}+\gamma+\delta+1\right) P_{n_{1}-1, n_{2}, n_{3}-1}^{(\alpha+1, \beta+1, \gamma+1, \delta+1)}(x, y, z) \\
& -\left(n_{1}+2 n_{2}+2 n_{3}+\beta+\gamma+\delta+2\right)\left(n_{2}+2 n_{3}+\gamma+\delta+1\right) \\
& \times\left(n_{3}+\gamma+\delta+1\right) P_{n_{1}, n_{2}-1, n_{3}-1}^{(\alpha+1, \beta+1, \gamma+1, \delta+1)}(x, y, z) .
\end{aligned}
$$

Proof The first relation comes from the equality

$$
\left(\mathcal{N}_{1,0} \mathcal{N}_{0,3}-\mathcal{N}_{0,5}^{+} \mathcal{N}_{6,0}^{+}\right) u=\left(2 n_{2}+2 n_{3}+\beta+\gamma+\delta+2\right)\left(\frac{\partial}{\partial x}-\frac{\partial}{\partial y}\right) u
$$

when $a=b=0$. Equation (27) is obtained when $a=b=0$ since

$$
\left(\mathcal{O}_{5,0}^{+} \mathcal{N}_{0,6}^{+}-\mathcal{N}_{0,1} \mathcal{O}_{3,0}\right) u=\left(2 n_{3}+\gamma+\delta+1\right)\left(\frac{\partial}{\partial z}-\frac{\partial}{\partial y}\right) u
$$

The relation given by $\mathcal{O}_{1,0}$ in Theorem 1 for $a=b=0$ gives (28). Finally, for the last relation, it is enough to combine the first and the third relations.

The derivatives of weighted versions of $P_{n_{1}, n_{2}, n_{3}}^{(\alpha, \beta, \gamma, \delta)}(x, y, z)$ verify the following sparse recurrence relations.

\section{Corollary 7 The following relations hold true:}

$$
\begin{aligned}
&\left(2 n_{2}+\right.\left.2 n_{3}+\beta+\gamma+\delta+2\right)\left(\frac{\partial}{\partial x}-\frac{\partial}{\partial y}\right)\left(x^{\alpha} y^{\beta} z^{\gamma} w^{\delta} P_{n_{1}, n_{2}, n_{3}}^{(\alpha, \beta, \gamma, \delta)}(x, y, z)\right) \\
&= x^{\alpha-1} y^{\beta-1} z^{\gamma} w^{\delta}\left\{\left(n_{1}+\alpha\right)\left(n_{2}+1\right) P_{n_{1}, n_{2}+1, n_{3}}^{(\alpha-1, \beta-1, \gamma)}(x, y, z)\right. \\
&\left.-\left(n_{1}+1\right)\left(n_{2}+\beta\right) P_{n_{1}+1, n_{2}, n_{3}}^{(\alpha-1, \beta-1, \delta)}(x, y, z)\right\}, \\
&\left(2 n_{3}+\gamma+\delta+1\right)\left(\frac{\partial}{\partial z}-\frac{\partial}{\partial y}\right)\left(x^{\alpha} y^{\beta} z^{\gamma} w^{\delta} P_{n_{1}, n_{2}, n_{3}}^{(\alpha, \beta, \gamma, \delta)}(x, y, z)\right) \\
&=x^{\alpha} y^{\beta-1} z^{\gamma-1} w^{\delta}\left\{-\left(n_{2}+\beta\right)\left(n_{3}+1\right) P_{n_{1}, n_{2}, n_{3}+1}^{(\alpha, \beta-1, \gamma-1)}(x, y, z)\right. \\
&\left.\quad+\left(n_{2}+1\right)\left(n_{3}+\gamma\right) P_{n_{1}, n_{2}+1, n_{3}}^{(\alpha, \beta-1, \gamma)}(x, y, z)\right\}, \\
& \frac{\partial}{\partial z}\left(x^{\alpha} y^{\beta} z^{\gamma} w^{\delta} P_{n_{1}, n_{2}, n_{3}}^{(\alpha, \beta, \gamma, \delta)}(x, y, z)\right)=-x^{\alpha} y^{\beta} z^{\gamma-1} w^{\delta-1}\left(n_{3}+1\right) P_{n_{1}, n_{2}, n_{3}+1}^{(\alpha, \beta, \gamma-1, \delta-1)}(x, y, z),
\end{aligned}
$$

and

$$
\begin{aligned}
\left(2 n_{2}\right. & \left.+2 n_{3}+\beta+\gamma+\delta+2\right) \frac{\partial}{\partial z}\left(\frac{\partial}{\partial x}-\frac{\partial}{\partial y}\right)\left(x^{\alpha} y^{\beta} z^{\gamma} w^{\delta} P_{n_{1}, n_{2}, n_{3}}^{(\alpha, \beta)}(x, y, z)\right) \\
= & -x^{\alpha-1} y^{\beta-1} z^{\gamma-1} w^{\delta-1}\left\{\left(n_{1}+\alpha\right)\left(n_{2}+1\right)\left(n_{3}+1\right) P_{n_{1}, n_{2}+1, n_{3}+1}^{(\alpha-1, \beta-1, \gamma-1, \delta-1)}(x, y, z)\right. \\
& \left.-\left(n_{1}+1\right)\left(n_{2}+\beta\right)\left(n_{3}+1\right) P_{n_{1}+1, n_{2}, n_{3}+1}^{(\alpha-1, \beta-1, \gamma-1, \delta-1)}(x, y, z)\right\} .
\end{aligned}
$$


Proof The first relation is a consequence of

$$
\begin{aligned}
& \left(\mathcal{N}_{1,0}^{+} \mathcal{N}_{0,3}^{+}-\mathcal{N}_{0,5} \mathcal{N}_{6,0}\right) u \\
& =\left(2 n_{2}+2 n_{3}+\beta+\gamma+\delta+2\right)\left(x y\left(\frac{\partial}{\partial y}-\frac{\partial}{\partial x}\right)+\beta x-\alpha y\right) u \\
& =-\left(2 n_{2}+2 n_{3}+\beta+\gamma+\delta+2\right) x^{1-\alpha} y^{1-\beta} w^{-\delta}\left(\frac{\partial}{\partial x}-\frac{\partial}{\partial y}\right)\left(x^{\alpha} y^{\beta} w^{\delta} u\right)
\end{aligned}
$$

The second one comes from the relation

$$
\begin{aligned}
\left(\mathcal{O}_{5,0} \mathcal{N}_{0,6}-\mathcal{N}_{0,1}^{+} \mathcal{O}_{3,0}^{+}\right) u & =\left(2 n_{3}+\gamma+\delta+1\right)\left(y z\left(\frac{\partial}{\partial y}-\frac{\partial}{\partial z}\right)+\beta z-\gamma y\right) u \\
& =-\left(2 n_{3}+\gamma+\delta+1\right) y^{1-\beta} z^{1-\gamma} w^{-\delta}\left(\frac{\partial}{\partial z}-\frac{\partial}{\partial y}\right)\left(y^{\beta} z^{\gamma} w^{\delta} u\right)
\end{aligned}
$$

The third relation holds from the relation

$$
\mathcal{O}_{1,0}^{+} u=(z \delta-w \gamma) u-z w \frac{\partial u}{\partial z}=-z^{1-\gamma} w^{1-\delta} \frac{\partial}{\partial z}\left(z^{\gamma} w^{\delta} u\right) .
$$

By taking into account the first and the third relations, we obtain the last relation.

It is possible to write $x P_{n_{1}, n_{2}, n_{3}}^{(\alpha, \beta, \gamma)}(x, y, z), \quad y P_{n_{1}, n_{2}, n_{3}}^{(\alpha, \beta, \gamma, \delta)}(x, y, z), \quad z P_{n_{1}, n_{2}, n_{3}}^{(\alpha, \beta, \gamma, \delta}(x, y, z)$, and $w P_{n_{1}, n_{2}, n_{3}}^{(\alpha, \beta, \gamma}(x, y, z)$ in terms of Koornwinder polynomials with different parameters as follows.

\section{Corollary 8 The following relations are satisfied:}

$$
\begin{aligned}
(2 n+ & \alpha+\beta+\gamma+\delta+3) x P_{n_{1}, n_{2}, n_{3}}^{(\alpha, \beta, \gamma, \delta)}(x, y, z) \\
= & \left(n_{1}+\alpha\right) P_{n_{1}, n_{2}, n_{3}}^{(\alpha,-\beta, \gamma)}(x, y, z)+\left(n_{1}+1\right) P_{n_{1}+1, n_{2}, n_{3}}^{(\alpha-1, \beta, \gamma, \delta)}(x, y, z), \\
(2 n+ & \alpha+\beta+\gamma+\delta+3)\left(2 n_{2}+2 n_{3}+\beta+\gamma+\delta+2\right) y P_{n_{1}, n_{2}, n_{3}}^{(\alpha, \beta, \gamma)}(x, y, z) \\
= & \left(n+n_{2}+n_{3}+\beta+\gamma+\delta+2\right)\left(n_{2}+\beta\right) P_{n_{1}, n_{2}, n_{3}}^{(\alpha, \beta-1, \gamma}(x, y, z) \\
& +\left(n+n_{2}+n_{3}+\alpha+\beta+\gamma+\delta+3\right)\left(n_{2}+1\right) P_{n_{1}, n_{2}+1, n_{3}}^{(\alpha, \beta-1, \gamma)}(x, y, z) \\
& -\left(n_{1}+1\right)\left(n_{2}+\beta\right) P_{n_{1}+1, n_{2}, n_{3}}^{(\alpha, \beta-1, \gamma)}(x, y, z)-\left(n_{1}+\alpha\right)\left(n_{2}+1\right) P_{n_{1}-1, n_{2}+1, n_{3}}^{(\alpha, \beta-1, \gamma, \delta)}(x, y, z), \\
(2 n+ & \alpha+\beta+\gamma+\delta+3)\left(2 n_{2}+2 n_{3}+\beta+\gamma+\delta+2\right)\left(\gamma+\delta+2 n_{3}+1\right) z P_{n_{1}, n_{2}, n_{3}}^{(\alpha, \beta, \gamma)}(x, y, z) \\
= & \left(n+n_{2}+n_{3}+\beta+\gamma+\delta+2\right)\left(n_{2}+2 n_{3}+\gamma+\delta+1\right)\left(n_{3}+\gamma\right) P_{n_{1}, n_{2}, n_{3}}^{(\alpha, \beta)}(x, y, z) \\
& -\left(n_{1}+1\right)\left(n_{2}+2 n_{3}+\gamma+\delta+1\right)\left(n_{3}+\gamma\right) P_{n_{1}+1, n_{2}, n_{3}}^{(\alpha, \beta, \gamma-1, \delta)}(x, y, z) \\
& -\left(n+n_{2}+n_{3}+\alpha+\beta+\gamma+\delta+3\right)\left(n_{2}+1\right)\left(n_{3}+\gamma\right) P_{n_{1}, n_{2}+1, n_{3}}^{(\alpha, \beta, \gamma-1, \delta)}(x, y, z) \\
& +\left(n_{1}+\alpha\right)\left(n_{2}+1\right)\left(n_{3}+\gamma\right) P_{n_{1}-1, n_{2}+1, n_{3}}^{(\alpha, \beta, \gamma-1, \delta)}(x, y, z) \\
& +\left(n+n_{2}+n_{3}+\alpha+\beta+\gamma+\delta+3\right)\left(n_{2}+2 n_{3}+\beta+\gamma+\delta+2\right)\left(n_{3}+1\right) \\
& \times P_{n_{1}, n_{2}, n_{3}+1}^{(\alpha, \beta, \gamma-1, \delta)}(x, y, z) \\
& -\left(n_{1}+\alpha\right)\left(n_{2}+2 n_{3}+\beta+\gamma+\delta+2\right)\left(n_{3}+1\right) P_{n_{1}-1, n_{2}, n_{3}+1}^{(\alpha, \beta, \gamma-1, \delta)}(x, y, z)
\end{aligned}
$$




$$
\begin{aligned}
& -\left(n+n_{2}+n_{3}+\beta+\gamma+\delta+2\right)\left(n_{2}+\beta\right)\left(n_{3}+1\right) P_{n_{1}, n_{2}-1, n_{3}+1}^{(\alpha, \beta, \gamma-1, \delta)}(x, y, z) \\
& +\left(n_{1}+1\right)\left(n_{2}+\beta\right)\left(n_{3}+1\right) P_{n_{1}+1, n_{2}-1, n_{3}+1}^{(\alpha, \beta, \gamma-1, \delta)}(x, y, z),
\end{aligned}
$$

and

$$
\begin{aligned}
(2 n+ & \alpha+\beta+\gamma+\delta+3)\left(2 n_{2}+2 n_{3}+\beta+\gamma+\delta+2\right)\left(\gamma+\delta+2 n_{3}+1\right) \\
& \times w P_{n_{1}, n_{2}, n_{3}}^{(\alpha, \beta, \gamma, y}(x, z) \\
= & \left(n+n_{2}+n_{3}+\alpha+\beta+\gamma+\delta+3\right)\left(n_{2}+2 n_{3}+\beta+\gamma+\delta+2\right)\left(n_{3}+1\right) \\
& \times P_{n_{1}, n_{2}, n_{3}+1}^{(\alpha, \beta, \gamma, \delta-1)}(x, y, z) \\
& +\left(n_{1}+\alpha\right)\left(n_{2}+2 n_{3}+\beta+\gamma+\delta+2\right)\left(n_{3}+1\right) P_{n_{1}-1, n_{2}, n_{3}+1}^{(\alpha, \beta, \gamma, \delta-1)}(x, y, z) \\
& +\left(n+n_{2}+n_{3}+\beta+\gamma+\delta+2\right)\left(n_{2}+\beta\right)\left(n_{3}+1\right) P_{n_{1}, n_{2}-1, n_{3}+1}^{(\alpha, \beta, \gamma, \delta-1)}(x, y) \\
& -\left(n_{1}+1\right)\left(n_{2}+\beta\right)\left(n_{3}+1\right) P_{n_{1}+1, n_{2}-1, n_{3}+1}^{(\alpha, \beta, \gamma, \delta-1)}(x, y, z) \\
& +\left(n+n_{2}+n_{3}+\beta+\gamma+\delta+2\right)\left(n_{2}+2 n_{3}+\gamma+\delta+1\right)\left(n_{3}+\delta\right) P_{n_{1}, n_{2}, n_{3}}^{(\alpha, \beta, \gamma, \delta}(x, y, z) \\
& -\left(n_{1}+1\right)\left(n_{2}+2 n_{3}+\gamma+\delta+1\right)\left(n_{3}+\delta\right) P_{n_{1}+1, n_{2}, n_{3}}^{(\alpha, \beta, \gamma, \delta-1)}(x, y, z) \\
& -\left(n+n_{2}+n_{3}+\alpha+\beta+\gamma+\delta+3\right)\left(n_{2}+1\right)\left(n_{3}+\delta\right) P_{n_{1}, n_{2}+1, n_{3}}^{(\alpha, \beta, \gamma, \delta-1)}(x, y, z) \\
& +\left(n_{1}+\alpha\right)\left(n_{2}+1\right)\left(n_{3}+\delta\right) P_{n_{1}-1, n_{2}+1, n_{3}}^{(\alpha, \beta, \gamma, \delta-1)}(x, y, z) .
\end{aligned}
$$

Proof The first relation follows from $\left(\mathcal{N}_{3,0}^{+}+\mathcal{N}_{5,0}\right) u=(2 n+\alpha+\beta+\gamma+\delta+3) x u$. The second relation is satisfied by the equality

$$
\begin{gathered}
(2 n+\alpha+\beta+\gamma+\delta+3)\left(2 n_{2}+2 n_{3}+\beta+\gamma+\delta+2\right) y u \\
=\mathcal{N}_{0,3}^{+}\left(\mathcal{N}_{2,0}^{+}-\mathcal{N}_{4,0}\right) u+\mathcal{N}_{0,5}\left(\mathcal{N}_{2,0}-\mathcal{N}_{4,0}^{+}\right) u
\end{gathered}
$$

The third relation comes from

$$
\begin{aligned}
& (2 n+\alpha+\beta+\gamma+\delta+3)\left(2 n_{2}+2 n_{3}+\beta+\gamma+\delta+2\right)\left(\gamma+\delta+2 n_{3}+1\right) z u \\
& \quad=\left(\mathcal{O}_{3,0}^{+} \mathcal{N}_{0,2}^{+}-\mathcal{O}_{5,0} \mathcal{N}_{0,4}^{+}\right)\left(\mathcal{N}_{2,0}^{+}-\mathcal{N}_{4,0}\right) u-\left(\mathcal{O}_{3,0}^{+} \mathcal{N}_{0,4}-\mathcal{O}_{5,0} \mathcal{N}_{0,2}\right)\left(\mathcal{N}_{2,0}-\mathcal{N}_{4,0}^{+}\right) u
\end{aligned}
$$

The last one is obtained from the relation

$$
\begin{aligned}
& (2 n+\alpha+\beta+\gamma+\delta+3)\left(2 n_{2}+2 n_{3}+\beta+\gamma+\delta+2\right)\left(\gamma+\delta+2 n_{3}+1\right) w u \\
& \quad=\left(\mathcal{O}_{4,0} \mathcal{N}_{0,4}^{+}+\mathcal{O}_{2,0}^{+} \mathcal{N}_{0,2}^{+}\right)\left(\mathcal{N}_{2,0}^{+}-\mathcal{N}_{4,0}\right) u-\left(\mathcal{O}_{4,0} \mathcal{N}_{0,2}+\mathcal{O}_{2,0}^{+} \mathcal{N}_{0,4}\right)\left(\mathcal{N}_{2,0}-\mathcal{N}_{4,0}^{+}\right) u
\end{aligned}
$$

\section{Generating functions for the polynomials with $2 d$-parameters on the d-dimensional simplex}

Using the generating functions of the classical univarite Jacobi polynomials, we can derive the generating functions for the polynomials $P_{\mathbf{n}}^{(\alpha)}(\mathbf{x})$ defined by (13) on the d-dimensional simplex. For this purpose, we first recall the following generating functions for the classical univariate Jacobi polynomials ([11], p. 82 (1) (2), p. 165 Problem 9):

$$
\sum_{n=0}^{\infty} P_{n}^{(\alpha, \beta)}(x) t^{n}=2^{\alpha+\beta} \rho^{-1}(1+t+\rho)^{-\beta}(1-t+\rho)^{-\alpha}, \quad|t|<1
$$




$$
\begin{aligned}
& \rho=\sqrt{1-2 t x+t^{2}} \\
& \sum_{n=0}^{\infty} P_{n}^{(\alpha, \beta-n)}(x) t^{n}=(1-t)^{\beta}\left(1-\frac{(x+1) t}{2}\right)^{-(\alpha+\beta+1)}, \quad|t|<\min \left\{1, \frac{2}{|x+1|}\right\}
\end{aligned}
$$

and

$$
\sum_{n=0}^{\infty} P_{n}^{(\alpha-n, \beta-n)}(x) t^{n}=\left(1+\frac{(x+1) t}{2}\right)^{\alpha}\left(1+\frac{(x-1) t}{2}\right)^{\beta}, \quad|t|<\min \left\{\frac{2}{|x+1|}, \frac{2}{|x-1|}\right\} .
$$

Theorem 9 The polynomials $P_{\mathbf{n}}^{\left(\alpha_{1}, \ldots, \alpha_{d+1}, \alpha_{d+2}-2 n_{2}-1, \ldots, \alpha_{2 d}-2 n_{d}-1\right)}(\mathbf{x})$ have a generating function as follows:

$$
\begin{aligned}
& \sum_{\mathbf{n}=\mathbf{0}}^{\infty} P_{\mathbf{n}}^{\left(\alpha_{1}, \ldots, \alpha_{d+1}, \alpha_{d+2}-2 n_{2}-1, \ldots, \alpha_{2 d^{-}}-2 n_{d}-1\right)}(\mathbf{x}) t^{|\mathbf{n}|} \\
& \quad=\prod_{j=1}^{d} 2^{a_{j}+\alpha_{j}} \rho_{j}^{-1}\left(1+t\left(1-\left|x_{j-1}\right|\right)+\rho_{j}\right)^{-\alpha_{j}}\left(1-t\left(1-\left|x_{j-1}\right|\right)+\rho_{j}\right)^{-a_{j}},
\end{aligned}
$$

for $\left|t\left(1-\left|x_{j-1}\right|\right)\right|<1(j=1,2, \ldots, d)$ where

$$
\begin{aligned}
& a_{j}=\left|\alpha^{j+1}\right|+\left|\widetilde{\alpha}^{d+j+1}\right|, \\
& \rho_{j}=\left(1-2 t\left(2 x_{j}+\left|x_{j-1}\right|-1\right)+t^{2}\left(1-\left|x_{j-1}\right|\right)^{2}\right)^{1 / 2}, \quad 1 \leq j \leq d .
\end{aligned}
$$

Proof If we use the definition of polynomials $P_{\mathbf{n}}^{(\boldsymbol{\alpha})}(\mathbf{x})$ given by (13) by taking $\alpha_{d+j+1} \rightarrow$ $\alpha_{d+j+1}-2 n_{j+1}-1,1 \leq j \leq d-1$, it follows from the generating function (38) that

$$
\begin{aligned}
& \sum_{\mathbf{n}=\mathbf{0}}^{\infty} P_{\mathbf{n}}^{\left(\alpha_{1}, \ldots, \alpha_{d+1}, \alpha_{d+2}-2 n_{2}-1, \ldots, \alpha_{2 d}-2 n_{d}-1\right)}(\mathbf{x}) t^{|\mathbf{n}|} \\
& \quad=\prod_{j=1}^{d}\left(\sum_{n_{j}=0}^{\infty}\left(\frac{1-\left|x_{j}\right|}{1-\left|x_{j-1}\right|}\right)^{\left|n^{j+1}\right|} P_{n_{j}}^{\left(a_{j}, b_{j}\right)}\left(\frac{2 x_{j}}{1-\left|x_{j-1}\right|}-1\right) t^{n_{j}}\right) \\
& =\prod_{j=1}^{d}\left(\sum_{n_{j}=0}^{\infty} P_{n_{j}}^{\left(a_{j}, b_{j}\right)}\left(\frac{2 x_{j}}{1-\left|x_{j-1}\right|}-1\right)\left(1-\left|x_{j-1}\right|\right)^{n_{j}} t^{n_{j}}\right) \\
& \quad=\prod_{j=1}^{d} 2^{a_{j}+b_{j}} \rho_{j}^{-1}\left(1+t\left(1-\left|x_{j-1}\right|\right)+\rho_{j}\right)^{-b_{j}}\left(1-t\left(1-\left|x_{j-1}\right|\right)+\rho_{j}\right)^{-a_{j}},
\end{aligned}
$$

for $\left|t\left(1-\left|x_{j-1}\right|\right)\right|<1(j=1,2, \ldots, d)$ where

$$
\begin{aligned}
& a_{j}=\left|\alpha^{j+1}\right|+\left|\widetilde{\alpha}^{d+j+1}\right|, \quad b_{j}=\alpha_{j}, \\
& \rho_{j}=\left(1-2 t\left(2 x_{j}+\left|x_{j-1}\right|-1\right)+t^{2}\left(1-\left|x_{j-1}\right|\right)^{2}\right)^{1 / 2}, \quad 1 \leq j \leq d .
\end{aligned}
$$


Corollary 10 In the case $d=3$, the polynomials $P_{n_{1}, n_{2}, n_{3}}^{\left(\alpha, \beta, 2, \beta n_{2}-1, b-2 n_{3}-1\right)}(x, y, z)$ with six parameters have a generating function as follows:

$$
\begin{aligned}
& \sum_{n_{1}, n_{2}, n_{3}=0}^{\infty} P_{n_{1}, n_{2}, n_{3}}^{\left(\alpha, \beta, \gamma, a-2 n_{2}-1, b-2 n_{3}-1\right)}(x, y, z) t^{n_{1}+n_{2}+n_{3}} \\
& =2^{\alpha+2 \beta+3 \gamma+3 \delta+a+2 b} \rho_{1}^{-1} \rho_{2}^{-1} \rho_{3}^{-1}\left(1+t+\rho_{1}\right)^{-\alpha}\left(1-t+\rho_{1}\right)^{-(\beta+\gamma+\delta+a+b)} \\
& \quad \times\left(1+t(1-x)+\rho_{2}\right)^{-\beta}\left(1-t(1-x)+\rho_{2}\right)^{-(\gamma+\delta+b)} \\
& \quad \times\left(1+t(1-x-y)+\rho_{3}\right)^{-\gamma}\left(1-t(1-x-y)+\rho_{3}\right)^{-\delta},
\end{aligned}
$$

for $|t|<\min \left\{1, \frac{1}{1-x}, \frac{1}{1-x-y}\right\}$ where

$$
\begin{aligned}
& \rho_{1}=\left(1-2 t(2 x-1)+t^{2}\right)^{1 / 2}, \\
& \rho_{2}=\left(1-2 t(2 y+x-1)+t^{2}(1-x)^{2}\right)^{1 / 2}, \\
& \rho_{3}=\left(1-2 t(2 z+x+y-1)+t^{2}(1-x-y)^{2}\right)^{1 / 2} .
\end{aligned}
$$

Theorem 11 The polynomials $P_{\mathbf{n}}^{\left(\alpha_{1}-n_{1}, \alpha_{2}, \ldots, \alpha_{d+1}, \alpha_{d+2}-2 n_{2}-1, \ldots, \alpha_{2 d^{-}}-2 n_{d}-1\right)}(\mathbf{x})$ are generated by

$$
\begin{aligned}
& \sum_{\mathbf{n}=\mathbf{0}}^{\infty} P_{\mathbf{n}}^{\left(\alpha_{1}-n_{1}, \alpha_{2}, \ldots, \alpha_{d+1}, \alpha_{d+2}-2 n_{2}-1, \ldots, \alpha_{2 d}-2 n_{d}-1\right)}(\mathbf{x}) t^{|\mathbf{n}|} \\
& =\prod_{j=2}^{d} 2^{a_{j}+\alpha_{j}} \rho_{j}^{-1}\left(1+t\left(1-\left|x_{j-1}\right|\right)+\rho_{j}\right)^{-\alpha_{j}}\left(1-t\left(1-\left|x_{j-1}\right|\right)+\rho_{j}\right)^{-a_{j}} \\
& \quad \times(1-t)^{\alpha_{1}}\left(1-t x_{1}\right)^{-(|\alpha|+1)}
\end{aligned}
$$

for $\left|t\left(1-\left|x_{j-1}\right|\right)\right|<1(j=2,3, \ldots, d)$ and $|t|<\min \left\{1, \frac{1}{x_{1}}\right\}$ where $|\boldsymbol{\alpha}|=\alpha_{1}+\cdots+\alpha_{2 d}$ and, $a_{j}$ and $\rho_{j}$ are defined as in (41).

Proof By taking $\alpha_{1} \rightarrow \alpha_{1}-n_{1} ; \alpha_{d+j+1} \rightarrow \alpha_{d+j+1}-2 n_{j+1}-1,1 \leq j \leq d-1$ in the definition of the polynomials $P_{\mathbf{n}}^{(\boldsymbol{\alpha})}(\mathbf{x})$, given by (13), we can write

$$
\begin{aligned}
& \sum_{\mathbf{n}=\mathbf{0}}^{\infty} P_{\mathbf{n}}^{\left(\alpha_{1}-n_{1}, \alpha_{2}, \ldots, \alpha_{d+1}, \alpha_{d+2}-2 n_{2}-1, \ldots, \alpha_{2 d}-2 n_{d}-1\right)}(\mathbf{x}) t^{|\mathbf{n}|} \\
& \quad=\prod_{j=1}^{d}\left(\sum_{n_{j}=0}^{\infty}\left(\frac{1-\left|x_{j}\right|}{1-\left|x_{j-1}\right|}\right)^{\left|n^{j+1}\right|} P_{n_{j}}^{\left(a_{j}, b_{j}\right)}\left(\frac{2 x_{j}}{1-\left|x_{j-1}\right|}-1\right) t^{n_{j}}\right) \\
& \quad=\prod_{j=2}^{d}\left(\sum_{n_{j}=0}^{\infty} P_{n_{j}}^{\left(a_{j}, b_{j}\right)}\left(\frac{2 x_{j}}{1-\left|x_{j-1}\right|}-1\right)\left(1-\left|x_{j-1}\right|\right)^{n_{j}} t^{n_{j}}\right) \sum_{n_{1}=0}^{\infty} P_{n_{1}}^{\left(a_{1}, \alpha_{1}-n_{1}\right)}\left(2 x_{1}-1\right) t^{n_{1}},
\end{aligned}
$$

and from (38) and (39) it follows that

$$
\begin{aligned}
= & \prod_{j=2}^{d} 2^{a_{j}+\alpha_{j}} \rho_{j}^{-1}\left(1+t\left(1-\left|x_{j-1}\right|\right)+\rho_{j}\right)^{-\alpha_{j}}\left(1-t\left(1-\left|x_{j-1}\right|\right)+\rho_{j}\right)^{-a_{j}} \\
& \times(1-t)^{\alpha_{1}}\left(1-t x_{1}\right)^{-(|\alpha|+1)}
\end{aligned}
$$


for $\left|t\left(1-\left|x_{j-1}\right|\right)\right|<1(j=2,3, \ldots, d)$ and $|t|<\min \left\{1, \frac{1}{x_{1}}\right\}$ where $a_{j}$ and $\rho_{j}$ are given by (41).

Corollary 12 In the case $d=3$, the polynomials $P_{n_{1}, n_{1}, n_{3}}^{\left(\alpha-\gamma, n_{3}, n_{2}-2 n_{2}-1, b-2 n_{3}-1\right)}(x, y, z)$ with six parameters are generated by

$$
\begin{aligned}
\sum_{n_{1}, n_{2}, n_{3}=0}^{\infty} P_{n_{1}, n_{2}, n_{3}}^{\left(\alpha-n_{1}, \gamma, \delta, a-2 n_{2}-1, b-2 n_{3}-1\right)}(x, y, z) t^{n_{1}+n_{2}+n_{3}} \\
=2^{\beta+2 \gamma+2 \delta+b}(1-t)^{\alpha}(1-t x)^{-(\alpha+\beta+\gamma+\delta+a+b+1)} \rho_{2}^{-1} \rho_{3}^{-1} \\
\quad \times\left(1+t(1-x)+\rho_{2}\right)^{-\beta}\left(1-t(1-x)+\rho_{2}\right)^{-(\gamma+\delta+b)} \\
\quad \times\left(1+t(1-x-y)+\rho_{3}\right)^{-\gamma}\left(1-t(1-x-y)+\rho_{3}\right)^{-\delta},
\end{aligned}
$$

for $|t|<\min \left\{1, \frac{1}{x}, \frac{1}{1-x}, \frac{1}{1-x-y}\right\}$ where $\rho_{2}$ and $\rho_{3}$ are as in $(42)$.

Theorem 13 For the polynomials $P_{\mathbf{n}}^{\left(\alpha_{1}-n_{1}, \ldots, \alpha_{d}-n_{d}, \alpha_{d+1}, \alpha_{d+2}-n_{2}-1, \alpha_{d+3}-n_{3}-1, \ldots, \alpha_{2 d^{-}} n_{d}-1\right)}(\mathbf{x})$, a generating function is given by

$$
\sum_{\mathbf{n}=0}^{\infty} P_{\mathbf{n}}^{\left(\alpha_{1}-n_{1}, \ldots, \alpha_{d}-n_{d}, \alpha_{d+1}, \alpha_{d+2}-n_{2}-1, \alpha_{d+3}-n_{3}-1, \ldots, \alpha_{2 d}-n_{d}-1\right)}(\mathbf{x}) t^{|\mathbf{n}|}=\prod_{j=1}^{d} \frac{\left(1-t\left(1-\left|x_{j-1}\right|\right)\right)^{\alpha_{j}}}{\left(1-t x_{j}\right)^{\left|\alpha^{j}\right|+\left|\widetilde{\alpha}^{d+j+1}\right|+1}},
$$

for $|t|<\min \left\{\frac{1}{1-\left|x_{j-1}\right|}, \frac{1}{x_{j}}\right\}, j=1,2, \ldots, d$.

Proof If we get $\alpha_{i} \rightarrow \alpha_{i}-n_{i}, 1 \leq i \leq d ; \alpha_{d+j+1} \rightarrow \alpha_{d+j+1}-n_{j+1}-1,1 \leq j \leq d-1$ in the definition of $P_{\mathbf{n}}^{(\alpha)}(\mathbf{x})$, from (39) we obtain the desired generating function.

Corollary 14 For the case $d=3$, the polynomials $P_{n_{1}, n_{2}, n_{3}}^{\left(\alpha-n_{1}, \gamma-n_{2}, \gamma-n_{3}, \delta, a-n_{2}-1, b-n_{3}-1\right)}(x, y, z)$ with six parameters are generated by

$$
\begin{gathered}
\sum_{n_{1}, n_{2}, n_{3}=0}^{\infty} P_{n_{1}, n_{2}, n_{3}}^{\left(\alpha-n_{2}, \beta-\gamma-n_{3}, \delta, a-n_{2}-1, b-n_{3}-1\right)}(x, y, z) t^{n_{1}+n_{2}+n_{3}} \\
=\frac{(1-t)^{\alpha}(1-t(1-x))^{\beta}(1-t(1-x-y))^{\gamma}}{(1-t x)^{\alpha+\beta+\gamma+\delta+a+b+1}(1-t y)^{\beta+\gamma+\delta+b+1}(1-t z)^{\gamma+\delta+1}},
\end{gathered}
$$

for $|t|<\min \left\{1, \frac{1}{1-x}, \frac{1}{1-x-y}, \frac{1}{x}, \frac{1}{y}, \frac{1}{z}\right\}$.

Theorem 15 The polynomials $P_{\mathbf{n}}^{\left(\alpha_{1}-n_{1}, \ldots, \alpha_{d}-n_{d}, \alpha_{d+1}-n_{d}, \alpha_{d+2}-n_{1}-1, \alpha_{d+3}-n_{2}-1, \ldots, \alpha_{2 d}-n_{d-1}-1\right)}(\mathbf{x})$ are generated by

$$
\begin{gathered}
\sum_{\mathbf{n}=0}^{\infty} P_{\mathbf{n}}^{\left(\alpha_{1}-n_{1}, \ldots, \alpha_{d}-n_{d}, \alpha_{d+1}-n_{d}, \alpha_{d+2}-n_{1}-1, \alpha_{d+3}-n_{2}-1, \ldots, \alpha_{2 d}-n_{d-1}-1\right)}(\mathbf{x}) t^{|\mathbf{n}|} \\
=\prod_{j=1}^{d}\left(1+t x_{j}\right)^{\left|\alpha^{j+1}\right|+\left|\widetilde{\alpha}^{d+j+1}\right|}\left(1+t\left(x_{j}+\left|x_{j-1}\right|-1\right)\right)^{\alpha_{j}},
\end{gathered}
$$

for $|t|<\min \left\{\frac{1}{x_{j}}, \frac{1}{x_{j}+\left|x_{j-1}\right|-1}\right\}, j=1,2, \ldots, d$. 
Proof It is enough to take $\alpha_{i} \rightarrow \alpha_{i}-n_{i}, 1 \leq i \leq d ; \alpha_{d+1} \rightarrow \alpha_{d+1}-n_{d} ; \alpha_{d+j+1} \rightarrow \alpha_{d+j+1}-n_{j}-1$, $1 \leq j \leq d-1$ in the definition of $P_{\mathbf{n}}^{(\alpha)}(\mathbf{x})$ and then use (40).

Corollary 16 In the case $d=3$, the polynomials $P_{n_{1}, n_{2}, n_{3}}^{\left(\alpha-n_{1}, \beta-n_{2}, \gamma-n_{3}, \delta-n_{3}, a-n_{1}-1, b-n_{2}-1\right)}(x, y, z)$ with six parameters are generated by

$$
\begin{gathered}
\sum_{n_{1}, n_{2}, n_{3}=0}^{\infty} P_{n_{1}, n_{2}, n_{3}}^{\left(\alpha-n_{1}, \beta-n_{2}, \gamma-n_{3}, \delta-n_{3}, a-n_{1}-1, b-n_{2}-1\right)}(x, y, z) t^{n_{1}+n_{2}+n_{3}} \\
=(1+t x)^{\beta+\gamma+\delta+a+b}(1+t y)^{\gamma+\delta+b}(1+t z)^{\delta} \\
\quad \times(1+t(x-1))^{\alpha}(1+t(y+x-1))^{\beta}(1+t(x+y+z-1))^{\gamma} \\
\text { for }|t|<\min \left\{\frac{1}{x}, \frac{1}{y}, \frac{1}{z}, \frac{1}{x-1}, \frac{1}{x+y-1}, \frac{1}{x+y+z-1}\right\} .
\end{gathered}
$$

\section{Acknowledgements}

The authors are grateful to both reviewers for helpful suggestions that improved the manuscript.

\section{Funding}

The research of R. Aktaş was done while she was visiting Universidade de Vigo, Spain, and the research has been supported by The Scientific and Technological Research Council of Turkey (TUBiTAK), Grant 1059B191802162. The work of the second author has been partially supported by the Agencia Estatal de Investigación (AEI) of Spain under Grant MTM2016-75140-P, and was cofinanced by the European Community fund FEDER.

\section{Availability of data and materials}

Not applicable (data sharing not applicable to this article as no data sets were generated or analyzed during the current study).

\section{Competing interests}

The authors declare that they have no competing interests.

\section{Authors' contributions}

The authors contributed equally and significantly in writing this paper. All authors read and approved the final manuscript.

\section{Author details}

'Department of Mathematics, Faculty of Science, Ankara University, Ankara, Turkey. ${ }^{2}$ Departamento de Matemática Aplicada II, E.E. Aeronáutica e do Espazo, Universidade de Vigo, Ourense, Spain. ${ }^{3}$ Department of Mathematics, Atilim University, Ankara, Turkey.

\section{Publisher's Note}

Springer Nature remains neutral with regard to jurisdictional claims in published maps and institutional affiliations.

Received: 7 February 2020 Accepted: 5 June 2020 Published online: 16 June 2020

\section{References}

1. Area, l., Godoy, E., Ronveaux, A., Zarzo, A.: Bivariate second-order linear partial differential equations and orthogonal polynomial solutions. J. Math. Anal. Appl. 387(2), 1188-1208 (2012)

2. Askey, R.: Orthogonal Polynomials and Special Functions. CBMS Regional Conf. Ser. in Appl. Math., vol. 21. SIAM, Philadelphia (1975)

3. Dunkl, C.F., Xu, Y.: Orthogonal Polynomials of Several Variables. Cambridge University Press, New York (2001)

4. Ismail, M.E.H.: Classical and Quantum Orthogonal Polynomials in One Variable. Encylopedia Math. Appl., vol. 98. Cambridge University Press, Cambridge (2005)

5. Koepf, W., Schmersau, D.: Representations of orthogonal polynomials. J. Comput. Appl. Math. 90, 57-94 (1998)

6. Koornwinder, T.H.: Two variable analogues of the classical orthogonal polynomials. In: Askey, R.A. (ed.) Theory and Application of Special Functions. Proceedings of an Advanced Seminar, pp. 435-495. Academic Press, New York (1975)

7. Lewanowicz, S.: The hypergeometric functions approach to the connection problem for the classical orthogonal polynomials. Technical report, Inst. of Computer Sci., Univ. of Wroclaw (2003)

8. Luke, Y.L.: The Special Functions and Their Approximations, vol. II. Academic Press, New York (1969)

9. Olver, S., Townsend, A., Vasil, G.M.: Recurrence relations for a family of orthogonal polynomials on a triangle. In: Spectral and High Order Methods for Partial Differential Equations ICOSAHOM 2018. Lecture Notes in Computational Science and Engineering, vol. 134. Springer, Berlin (2020). arXiv:1801.09099 
10. Rainville, E.D.: Special Functions. Macmillan, New York (1960)

11. Srivastava, H.M., Manocha, H.L.: A Treatise on Generating Functions. Halsted Press, Chichester, Wiley, New York (1984)

12. Suetin, P.K.: Orthogonal Polynomials in Two Variables. Gordon \& Breach, Moscow (1988)

13. Szegö, G.: Orthogonal Polynomials. Amer. Math. Soc. Colloq. Publ., vol. 23, 4th edn. Am. Math. Soc., Providence (1975)

14. Zarzo, A.: Ecuaciones Diferenciales de Tipo Hipergeométrico Propiedades algebraicas y espectrales de sus soluciones. Aplicaciones Mecano-Cuánticas. Ph.D. thesis, Universidad de Granada (1995)

Submit your manuscript to a SpringerOpen ${ }^{\odot}$ journal and benefit from:

- Convenient online submission

Rigorous peer review

- Open access: articles freely available online

- High visibility within the field

- Retaining the copyright to your article

Submit your next manuscript at $\gg$ springeropen.com 